\title{
Improving Tomato Productivity under High Temperature Conditions
}

\author{
El-Tantawy, E. M. ${ }^{* 1}$ and Rewaa S. El-Shatoury ${ }^{2}$ \\ ${ }^{1}$ Plant Production Department (Vegetables), Fac. Environ. Agric. Sci., Arish Univ., Egypt. \\ ${ }^{2}$ Horticultural Department (Vegetables), Agric. Fac., Suez Canal Univ., Egypt.
}

Received: $8 / 3 / 2017$

\begin{abstract}
Two field experiments were carried out during the summer seasons of 2014 and 2015 at the Experimental Farm of the Faculty of Environmental Agricultural Sciences, El-Arish, Arish University, North Sinai Governorate, Egypt to improve the performance of tomato plants (Hybrid Alissa F1) grown under high temperature. This study included 12 treatments which were the combination between application of two types of floating covers (bare plants, covering with Agryl) and spraying with some growth substances to improve the fruit setting under heat stress (control, $\left.\mathrm{Ca}+\mathrm{B}, \mathrm{GA}_{3}, \mathrm{IAA}, \mathrm{GA}_{3}+\mathrm{Ca}+\mathrm{B}, \mathrm{IAA}+\mathrm{Ca}+\mathrm{B}\right) ; \mathrm{Ca}, \mathrm{B}, \mathrm{GA}_{3}$, and IAA were used at 500, 25, 20 and 20 ppm, respectively. The results showed that tomato plant growth (both of fresh weight and dry weight) proline concentration, and fruit setting were increased with application of floating cover (Agryl), while total chlorophyll content, marketable yield per plant and per feddan, total yield/fed. and number of seeds/fruit were decreased. Spraying with $I_{A A}, G_{3}+C a+B$ and $\mathrm{IAA}+\mathrm{Ca}+\mathrm{B}$ were the best treatments for total dry weight, total chlorophyll, proline and fruit setting while IAA $+\mathrm{Ca}+\mathrm{B}$ was the best treatment for both marketable yield /plant and per fed., total yield/fed. as well as number of seeds/fed. The best interaction treatments for total chlorophyll, the components of marketable yield and number of seeds/fruit were spraying bare plantswithGA $\mathrm{A}_{3}+\mathrm{Ca}+\mathrm{B}$ or $\mathrm{IAA}+\mathrm{Ca}+\mathrm{B}$, while the same treatments under floating cover increased proline concentration in leaves and partially increased average fruit setting (\%).
\end{abstract}

Keywords: Tomato, high temperature, fruit set, chlorophyll, proline, yield

\section{INTRODUCTION}

Tomato (Solanum lycopersicum Mill.) is one of the most popular vegetable crops consumed all over the world. It is an important source of minerals, vitamins, and it has good amounts of lycopene and antioxidants which protect human body from free radicals and consequently reducing cancer disease. Tomato production has a close relation with the environmental conditions, especially air temperature. One of the methods used to modify the air condition around tomato plants is application of floating covers or spunbonded which is made from fine mesh of white synthetic fibers (polypropylene) as defined by Gordon (2006) and Taber and Webb (2008). Floating covers used to protect tomato plants from the infection of insects. Covering tomato plants by Agryl decreased the impact of tomato yellow leaf curl disease virus (TYLCV) (Berlinger et al., 2002; Al-Shihi et al., 2016). Many researchers used the floating covers to rise and study the higher temperature effects on behavior of tomato plants. Heat stress had low effect on vegetative growth, but it has negative activities of ROS enzymes (release oxygen scavenging) as SOD (Superoxide dismutase) and APX (Ascorbate peroxidase) which affect $\mathrm{CO}_{2}$ fixation negatively, viability of pollen grains and pollen tube growth which twist and grow in helical form (Pressman et al., 2002; Kafizadeh et al., 2008; Hu et al., 2010; Zhou et al., 2016) and consequently decrease the productivity of plant. Under these conditions plants try to overcome the harmful effects through producing a unique set of amino acids such as proline or chaperone proteins adverted to heat shock protein (HSPs) (Verbruggen and Hermans, 2008; Ördog, 2011). With the recent changes in environmental conditions and greenhouse phenomenon, some attempts were done to study or help plants to stand against heat stress effects such as spraying with nutrients such as Ca or B. Calcium or boron can enhance growth, fruit set and productivity of tomato plant separately but they are more effective with combinations (Asad et al., 2003). In addition application of auxin onto stigma promote pollen grain and ovary to develop (de Jong et al., 2009; Ördog, 2011). Therefore, this attempt was done to improve the performance of tomato plants under high temperature by application of some nutrients as $\mathrm{Ca}+\mathrm{B}$ and $\mathrm{GA}_{3}$ or IAA and their combinations as enhancing substances for fruit setting and yield production.

\section{MATERIALS AND METHODS}

Two field experiments were carried out during the summer seasons of 2014 and 2015 at the Experimental Farm of the Faculty of Environmental Agricultural Sciences, El-Arish, Arish University, North Sinai Governorate, Egypt to study the performance of tomato plants (Hybrid Alissa F1) grown under high temperature with application of floating covers (Agryl) and spraying with $\mathrm{Ca}, \mathrm{B}$, and some growth substances and their combinations to improve the fruit setting under heat stress. Some physico-chemical properties of the experimental soil sample which was taken at 0-30 cm depth are shown in Table (1) and chemical analysis of irrigation water is shown in Table (2).

The experiment included 12 treatments which were the interaction between two factors:

Factor A: application of floating covers; viz, bare plants (without application of cover) and application of spunbonded sheets (Agryl).

Factor B: contained 6 treatments which were spraying with $\mathrm{Ca}+\mathrm{B}$ and some growth substances to improve fruit setting as follows:

1- Control treatment (spraying with tap water)

2- Spraying with $\mathrm{Ca}+\mathrm{B}$

3- Spraying withGA 3

4- Spraying with IAA

5- Spraying with $\mathrm{GA}_{3}+\mathrm{Ca}+\mathrm{B}$

6- Spraying with IAA $+\mathrm{Ca}+\mathrm{B}$ 
Table (1): Initial soil physical and chemical analysis

\begin{tabular}{|c|c|c|c|}
\hline \multicolumn{2}{|c|}{ Soil properties } & First season (2014) & Second season (2015) \\
\hline \multicolumn{4}{|c|}{ Mechanical analysis } \\
\hline \multicolumn{2}{|c|}{ Soil texture } & Sandy & Sandy \\
\hline \multicolumn{4}{|c|}{ Chemical analysis (soluble ions in (1:5) extract) } \\
\hline $\mathrm{Ca}^{++}$ & $\left(\right.$meq..$\left.^{-1}\right)$ & 2.78 & 3.06 \\
\hline $\mathbf{M g}^{++}$ & $\left(\right.$meq..$\left.^{-1}\right)$ & 2.11 & 2.57 \\
\hline $\mathbf{N a}^{+}$ & $\left(\right.$meq..$\left.^{-1}\right)$ & 1.91 & 2.09 \\
\hline $\mathbf{K}^{+}$ & $\left(\right.$meq..$\left.^{-1}\right)$ & 0.41 & 0.49 \\
\hline $\mathrm{CO}_{3}^{--}$ & $\left(\right.$meq..$\left.^{-1}\right)$ & - & - \\
\hline $\mathrm{HCO}_{3}^{-}$ & $\left(\right.$meq..$\left.^{-1}\right)$ & 2.36 & 2.61 \\
\hline $\mathrm{Cl}^{-}$ & $\left(\right.$meq..$\left.^{-1}\right)$ & 1.65 & 1.89 \\
\hline $\mathrm{SO}_{4}^{--}$ & $\left(\right.$meq..$\left.^{-1}\right)$ & 3.18 & 3.40 \\
\hline \multicolumn{2}{|c|}{ Available N (ppm) } & 16.52 & 16.24 \\
\hline \multicolumn{2}{|c|}{ Available P (ppm) } & 46.50 & 45.21 \\
\hline \multicolumn{2}{|c|}{ Available K (ppm) } & 97.50 & 96.25 \\
\hline \multicolumn{2}{|c|}{ 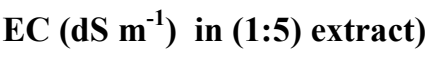 } & 0.75 & 0.79 \\
\hline \multicolumn{2}{|c|}{$\mathrm{pH}$ in $(1: 2.5)$ extract $)$} & 8.03 & 8.11 \\
\hline \multicolumn{2}{|c|}{$\mathrm{CaCO}_{3} \%$} & 9.85 & 10.98 \\
\hline
\end{tabular}

Table (2): Chemical analysis of irrigation water

\begin{tabular}{|c|c|c|c|c|c|c|c|c|c|c|}
\hline \multirow{3}{*}{ pH } & \multicolumn{2}{|c|}{$E C$} & \multicolumn{8}{|c|}{ Soluble ions $\left(\right.$ meq..$\left.^{-1}\right)$} \\
\hline & \multirow{2}{*}{$\mathrm{dSm}^{-1}$} & \multirow{2}{*}{ ppm } & \multicolumn{4}{|c|}{ Cations } & \multicolumn{4}{|c|}{ Anions } \\
\hline & & & $\mathrm{Ca}^{++}$ & $\mathrm{Mg}^{++}$ & $\mathrm{Na}^{+}$ & $\mathbf{K}^{+}$ & $\mathrm{Cl}^{-}$ & $\mathrm{HCO}_{3}^{-}$ & $\mathrm{CO}_{3}^{--}$ & $\mathrm{SO}_{4}^{--}$ \\
\hline \multicolumn{11}{|c|}{ First season (2014) } \\
\hline 7.22 & 6.12 & 3928.6 & 18.12 & 23.31 & 18.77 & 0.19 & 42.51 & 7.25 & - & 10.63 \\
\hline \multicolumn{11}{|c|}{ Second season (2015) } \\
\hline 7.05 & 5.98 & 3832.6 & 17.54 & 21.91 & 18.13 & 0.22 & 40.61 & 7.15 & - & 10.04 \\
\hline
\end{tabular}

Calcium was used with a concentration of 500 ppm, $\mathrm{B}$ at $25 \mathrm{ppm}, \mathrm{GA}_{3}$ at $20 \mathrm{ppm}$ and IAA at $20 \mathrm{ppm}$. Treatments were randomly arranged in split plot design with three replicates where the treatments of factor A were arranged in the main plots and the treatments of factor B were randomly arranged in the sub-plots. Calcium, $\mathrm{B}, \mathrm{GA}_{3}$, and IAA were added as foliar spray with application of super film $\left(1.0 \mathrm{ml} \mathrm{l}^{-1}\right)$ as spreading agent. Plants were sprayed with fruit setting substances three times with beginning of blooming (at 30, 40, and 50 days after transplanting). Seedlings were transplanted on $1^{\text {st }}$ may in both seasons. Seedlings were transplanted in single dripper lines with distances of 175 $\mathrm{cm}$ between the dripper lines and $50 \mathrm{~cm}$ between the transplants at the same dripper line. Plot area was 17.5 $\mathrm{m}^{2}$ (10 $\mathrm{m}$ in length and $175 \mathrm{~cm}$ in width). Plants were covered by floating covers (Agryl) at 21 days after transplanting on the frame of low tunnels and continued to the end of harvesting. Air temperatures and humidity inside and outside the tunnels during the two experimental seasons are shown in Table (3) which was registered daily in the midday (maximum value of temperature) by big screen indoor-outdoor thermo hygrometer.

All experimental units received compost at a rate of 4 tons/fed. (recommended dose). The source of compost was Al-Arabiah for organic fertilizer factory, Sharkia Governorate. Plants received the recommended dose of NPK $\left(120 \mathrm{~kg} \mathrm{~N}, 75 \mathrm{~kg} \mathrm{P}_{2} \mathrm{O}_{5}\right.$ and $120 \mathrm{~kg} \mathrm{k}_{2} \mathrm{O}$ /fed.). One third of NPK fertilizers quantity were added during soil preparation and the other two- thirds were divided into twenty portions and added gradually two times weekly through the irrigation water (fertigation) beginning eight days after transplanting. The other conventional practices were applied. 
Table (3): Air temperature and relative humidity without and under floating cover (Agryl) during 2014-2015 seasons

\begin{tabular}{|c|c|c|c|c|c|c|c|c|c|}
\hline \multirow[b]{2}{*}{ Month } & \multirow[b]{2}{*}{ Week } & \multicolumn{2}{|c|}{$\begin{array}{c}\text { Temperature } \\
\left({ }^{\circ} \mathrm{C}\right)\end{array}$} & \multicolumn{2}{|c|}{$\begin{array}{c}\text { Relative humidity } \\
(\%)\end{array}$} & \multicolumn{2}{|c|}{$\begin{array}{c}\text { Temperature } \\
\left({ }^{\circ} \mathrm{C}\right)\end{array}$} & \multicolumn{2}{|c|}{$\begin{array}{c}\text { Relative humidity } \\
(\%)\end{array}$} \\
\hline & & $\begin{array}{l}\text { Without } \\
\text { covering }\end{array}$ & $\begin{array}{c}\text { Floating } \\
\text { Cover } \\
\text { (Agryl) }\end{array}$ & $\begin{array}{l}\text { Without } \\
\text { covering }\end{array}$ & $\begin{array}{c}\text { Floating } \\
\text { Cover } \\
\text { (Agryl) } \\
\end{array}$ & $\begin{array}{l}\text { Without } \\
\text { covering }\end{array}$ & $\begin{array}{c}\text { Floating } \\
\text { Cover } \\
\text { (Agryl) } \\
\end{array}$ & $\begin{array}{l}\text { Without } \\
\text { covering }\end{array}$ & $\begin{array}{c}\text { Floating } \\
\text { Cover } \\
\text { (Agryl) }\end{array}$ \\
\hline & & \multicolumn{4}{|c|}{ First season (2014) } & \multicolumn{4}{|c|}{ Second season (2015) } \\
\hline \multirow{4}{*}{ June } & $1^{\text {st }}$ & 33.38 & 38.66 & 39.40 & 43.20 & 33.12 & 37.25 & 37.31 & 42.18 \\
\hline & $2^{\text {nd }}$ & 36.80 & 41.10 & 40.00 & 45.30 & 35.79 & 39.80 & 39.16 & 45.61 \\
\hline & $3^{\mathrm{rd}}$ & 35.20 & 38.90 & 42.25 & 46.33 & 36.31 & 40.23 & 41.05 & 46.13 \\
\hline & $4^{\text {th }}$ & 38.63 & 38.90 & 44.00 & 45.85 & 38.70 & 41.50 & 43.66 & 46.17 \\
\hline \multirow{2}{*}{ July } & $1^{\text {st }}$ & 39.83 & 40.66 & 44.33 & 46.33 & 38.97 & 41.77 & 44.17 & 46.81 \\
\hline & $2^{\text {nd }}$ & 38.60 & 41.90 & 43.50 & 46.15 & 38.71 & 40.98 & 43.33 & 46.63 \\
\hline
\end{tabular}

\section{Data recorded:}

Samples of three plants were randomly taken at 55- day after transplanting from each experimental unit to determine the following data:

1- Plant growth: It was estimated as:

- Plant height $(\mathrm{cm})$

- Number of both branches and leaves /plant

- Fresh weight of roots, stem, leaves and total fresh weight/plant (g)

- Dry weight of roots, stem, leaves, and total dry weight/plant (g)

- Total chlorophyll: Total chlorophyll was determined in the fourth leaf from tomato plant top using a digital chlorophyll meter, Minolta Chlorophyll Meter SPAD- 502, (Minolta Company, Japan).

2- Proline content in leaves was determined calorimetrically in leaves according to Bates et al. (1973).

3-Fruit setting (\%): Samples of five plants from each experimental unit were randomly chosen to estimate fruit setting (\%) in the $1^{\text {st }}$ three clusters of the main stem as following: Fruit setting $\%=\{$ (Total flowers cluster) / (total fruits/cluster) $\} \times 100$, and the average fruit setting (\%) of the three clusters was calculated.

4- Yield and its components: At red maturity stage, fruits of each plot were harvested, counted and weighed; the following data were recorded:

- Marketable yield: yield/plant (g) and yield/fed. (ton) were calculated,

- Unmarketable yield (ton/fed.) (infected fruits by blossom-end rot).

- Total yield (ton /fed.) was calculated as marketable yield (ton/fed.) + unmarketable yield (ton/fed.)

5- Seeds No./fruit (as indication for efficiency of pollination): Five fruits at red-ripe stage from each experimental unit were randomly taken, seeds were extracted, dried at room temperature, counted and average seeds number/fruit was calculated.

6- Statistical Analysis: The obtained data were subjected to statistical analysis of variance according to Snedecor and Cochran (1980). Duncan's multiple range test (1955) was used for means comparisons.

\section{RESULTS AND DISCUSSION}

\section{Plant growth}

\section{Effect of floating covers}

Data in Table (4) show significant effects of floating covers on tomato plant growth. The data revealed that covering tomato plants with Agryl increased all plant growth parameters, viz, plant height, number of branches and leaves/plant, fresh weight of roots, stem, leaves as well as total fresh weight of plant. The obtained results are true in both seasons.

\section{Effect of fruit setting substances}

Data in Table (4) show a significant increment in tomato plant height due to spraying with $\mathrm{Ca}+\mathrm{B}$ in both seasons, while number of both branches and leaves/plant were not significantly affected. The same data showed an augment in fresh weight of roots/ plant due to spraying tomato plants with IAA in the $1^{\text {st }}$ season, but it increased with spraying IAA $+\mathrm{Ca}+\mathrm{B}$ in the $2^{\text {nd }}$ season. Spraying with $\mathrm{GA}_{3}$ or IAA recorded the highest values of fresh weight of stem in the $1^{\text {st }}$ season, while spraying with $\mathrm{GA}_{3}+\mathrm{Ca}+\mathrm{B}$ was the superior treatment in the $2^{\text {nd }}$ one. In regard to fresh weight of leaves and total fresh weight of plant, the data revealed that spraying with $\mathrm{GA}_{3}, \mathrm{GA}_{3}+\mathrm{Ca}+\mathrm{B}$ and $\mathrm{IAA}+\mathrm{Ca}+\mathrm{B}$ recorded the highest values in the $1^{\text {st }}$ and $2^{\text {nd }}$ seasons, respectively.

It could be concluded that spraying tomato plants with $\mathrm{Ca}+\mathrm{B}$ alone or combined with $\mathrm{GA}_{3}$ or IAA were the best treatments for fresh weight of stem, leaves and total fresh weight/plant in the $1^{\text {st }}$ and $2^{\text {nd }}$ seasons, respectively. The increment in total fresh weight of plant may be due to the increase in fresh weight of stem and leaves per plant (Asad et al., 2003) and Rab and Haq (2012) found synergistic effect of $\mathrm{Ca}+\mathrm{B}$ combination. Boron increases IAA which regulates auxin supply by protecting the IAA oxidase system (Srivastava and Gupta, 1996). In addition, spraying $\mathrm{GA}_{3}$ promoted the vegetative growth of tomato plants that may be owe to the synergistic effect on DNA \& RNA proteins and polyribosome multiplication which increases leaf area of plant (Khan et al., 2006). 
Table (4): Effect of floating cover (Agryl) and fruit setting substances on tomato plant growth

\begin{tabular}{|c|c|c|c|c|c|c|c|}
\hline \multirow[b]{2}{*}{ Treatments } & \multicolumn{7}{|c|}{ Plant growth } \\
\hline & $\begin{array}{l}\text { Plant } \\
\text { height } \\
\text { (cm) }\end{array}$ & $\begin{array}{c}\text { No .of } \\
\text { branches } \\
\text { /plant }\end{array}$ & $\begin{array}{l}\text { No .of } \\
\text { leaves } \\
\text { /plant }\end{array}$ & $\begin{array}{l}\text { F.W. roots } \\
\text { /plant (g) }\end{array}$ & $\begin{array}{l}\text { F.W. stem } \\
\text { /plant (g) }\end{array}$ & $\begin{array}{c}\text { F.W. leaves } \\
\text { /plant (g) }\end{array}$ & $\begin{array}{c}\text { Total F.W. } \\
\text { /plant (g) }\end{array}$ \\
\hline \multicolumn{8}{|c|}{ First season (2014) } \\
\hline Bare plants & $62.58 \mathrm{~b}$ & $9.92 b$ & $57.33 b$ & $38.12 b$ & $93.20 \mathrm{~b}$ & $297.7 b$ & $428.9 b$ \\
\hline $\begin{array}{l}\text { Floating cover } \\
\text { (Agryl) }\end{array}$ & $73.66 \mathrm{a}$ & $13.58 \mathrm{a}$ & $70.33 a$ & $76.25 \mathrm{a}$ & $160.62 \mathrm{a}$ & $384.2 \mathrm{a}$ & $621.0 \mathrm{a}$ \\
\hline \multicolumn{8}{|c|}{ Second season (2015) } \\
\hline Bare plants & $64.22 b$ & $9.30 \mathrm{~b}$ & $59.33 b$ & $38.77 b$ & $91.83 b$ & $267.9 b$ & $398.5 b$ \\
\hline $\begin{array}{l}\text { Floating cover } \\
\text { (Agryl) }\end{array}$ & $73.77 \mathrm{a}$ & $12.21 \mathrm{a}$ & $68.05 \mathrm{a}$ & $65.43 \mathrm{a}$ & $142.77 \mathrm{a}$ & $313.2 \mathrm{a}$ & $521.4 \mathrm{a}$ \\
\hline \multicolumn{8}{|c|}{ First season (2014) } \\
\hline Control & $69.25 \mathrm{ab}$ & $10.25 \mathrm{a}$ & $59.50 \mathrm{a}$ & $32.38 \mathrm{c}$ & $109.8 \mathrm{~b}$ & $274.4 \mathrm{~d}$ & $416.5 d$ \\
\hline $\mathbf{C a}+\mathbf{B}$ & $76.75 \mathrm{a}$ & $11.50 \mathrm{a}$ & $67.75 \mathrm{a}$ & $42.50 \mathrm{c}$ & $128.3 \mathrm{ab}$ & $363.5 \mathrm{ab}$ & $534.3 \mathrm{bc}$ \\
\hline $\mathbf{G A}_{3}$ & $66.00 \mathrm{~b}$ & $12.25 \mathrm{a}$ & $62.75 \mathrm{a}$ & $68.25 b$ & $139.0 \mathrm{a}$ & $395.5 \mathrm{a}$ & $602.8 \mathrm{a}$ \\
\hline IAA & $67.75 \mathrm{ab}$ & $13.25 \mathrm{a}$ & $66.00 \mathrm{a}$ & $75.75 \mathrm{a}$ & $144.4 \mathrm{a}$ & $371.3 \mathrm{ab}$ & $591.4 \mathrm{ab}$ \\
\hline $\mathbf{G A}_{3}+\mathbf{C a}+\mathbf{B}$ & $67.00 \mathrm{~b}$ & $11.00 \mathrm{a}$ & $65.00 \mathrm{a}$ & $65.88 \mathrm{~b}$ & $109.9 \mathrm{~b}$ & $325.5 b c$ & $501.3 \mathrm{c}$ \\
\hline $\mathbf{I A A}+\mathbf{C a}+\mathbf{B}$ & $62.00 \mathrm{~b}$ & $12.25 \mathrm{a}$ & $62.00 \mathrm{a}$ & $58.38 b$ & $130.3 \mathrm{ab}$ & $315.3 \mathrm{~cd}$ & $503.9 c$ \\
\hline \multicolumn{8}{|c|}{ Second season (2015) } \\
\hline Control & $63.67 \mathrm{e}$ & $10.35 \mathrm{a}$ & $56.33 \mathrm{a}$ & $33.40 \mathrm{e}$ & $101.2 d$ & $254.3 \mathrm{c}$ & $388.8 \mathrm{~d}$ \\
\hline $\mathbf{C a}+\mathbf{B}$ & $73.67 \mathrm{a}$ & $10.00 \mathrm{a}$ & $64.83 \mathrm{a}$ & $40.15 d$ & $118.7 b$ & $293.0 \mathrm{~b}$ & $451.8 \mathrm{c}$ \\
\hline $\mathbf{G A}_{3}$ & $68.17 \mathrm{~cd}$ & $10.72 \mathrm{a}$ & $63.00 \mathrm{a}$ & $50.93 c$ & $122.8 \mathrm{ab}$ & $285.9 b$ & $459.7 \mathrm{c}$ \\
\hline IAA & $69.67 b c$ & $10.95 \mathrm{a}$ & $64.17 \mathrm{a}$ & $58.85 \mathrm{~b}$ & $121.7 \mathrm{ab}$ & $290.3 b$ & $470.8 b$ \\
\hline $\mathbf{G A}_{3}+\mathbf{C a}+\mathbf{B}$ & $71.67 \mathrm{ab}$ & $11.55 \mathrm{a}$ & $68.83 \mathrm{a}$ & $63.55 b$ & $125.7 \mathrm{a}$ & $309.4 a$ & $498.6 \mathrm{a}$ \\
\hline $\mathbf{I A A}+\mathbf{C a}+\mathbf{B}$ & $67.17 \mathrm{~d}$ & $11.00 \mathrm{a}$ & $65.00 \mathrm{a}$ & $65.75 \mathrm{a}$ & $113.8 \mathrm{c}$ & $310.5 \mathrm{a}$ & $490.1 \mathrm{a}$ \\
\hline
\end{tabular}

Values having the same alphabetical letter (s) did not significantly differ at 0.05 level of significance according to Duncan's multiple range test

\section{Effect of interaction between floating covers and fruit} setting substance

Data in Table (5) show significant effects of interaction between floating covers and fruit setting substances on tomato plant grown during high temperature season. The data showed that spraying tomato plants grown under floating cover (Agryl) with $\mathrm{Ca}+\mathrm{B}$ increased tomato plant height in both seasons. In addition, spraying with IAA under floating covers was the superior treatment, approximately for increasing all other traits; viz, number of both branches and leaves/plant, fresh weight of roots, stem, leaves and total fresh weight of plant in both seasons, except fresh weight of roots in the $2^{\text {nd }}$ season which increased with $\mathrm{IAA}+\mathrm{Ca}+\mathrm{B}$ foliar spray. These results may be due to the bioactive role of $\mathrm{Ca}$ and $\mathrm{B}$ for plant.Ca preserves the plant cell structure and resistance to different environmental stress (Mestre et al., 2012), Boron is an essential element for new cell structure and cell division through supplementing by sugars. Indol acetic acid plays an important role in enlargement of plant cells and promoting plant growth which affect the most fundamental responses of plant (Hopkins and Hüner, 2009). In this connection, Rab and Haq (2012) found increments in plant height and number of branches of tomato plants due to spraying $0.6 \% \mathrm{Ca}+0.2 \% \mathrm{~B}$ compared to application of $\mathrm{Ca}$ or $\mathrm{B}$ in single form or control treatment.

\section{Dry weight \\ Effect of floating covers}

The data in Table (6) illustrate that covering tomato plants with spun bonded sheets (Agryl) increased all traits of dry weight per plant expressed in dry weight of roots, stem, leaves and total dry weight in both seasons. The total dry weight due to application of 
Agryl was increased by $50.22 \%$ and $44.54 \%$ over that of the bare plants in the $1^{\text {st }}$ and $2^{\text {nd }}$ seasons, respectively. The increment in dry weight of different organs of tomato due to covering with spun bonded sheets may be owe to the increase in all plant growth traits as shown in Table (4). These results were coincide with Al-Shihi et al. (2016) who found increases in tomato plant growth and dry weight means of plant grown under floating row covers (Agryl).

\section{Effect of fruit setting substances}

It was obvious from the data in Table (6) that spraying tomato plants grown in high temperature seasons with fruit setting substances have significant effects on all dry weight parameters in both seasons.
Spraying tomato plants with IAA was the superior treatment in the $1^{\text {st }}$ season which increased all dry weight traits under study (dry weight of roots, stem, leaves and total dry weight/plant). On the other hand, spraying with $\mathrm{GA}_{3}+\mathrm{Ca}+\mathrm{B}$ recorded the highest value of total dry weight of plant as a result of increasing dry weight of stem and leaves followed by spraying IAA in the $2^{\text {nd }}$ season. Spraying IAA $+\mathrm{Ca}+\mathrm{B}$ increased dry weight of roots and leaves and consequently total dry weight, while spraying with IAA increased dry weight of both stem and leaves and then total dry weight of plant. Control treatment recorded the lowest values of above-mentioned parameters.

Table (5): Effect of interaction between floating cover (Agryl) and fruit setting substances on tomato plant growth

Plant growth parameters

\begin{tabular}{|c|c|c|c|c|c|c|c|c|}
\hline Treatments & & $\begin{array}{l}\text { Plant } \\
\text { height } \\
\text { (cm) }\end{array}$ & $\begin{array}{c}\text { No. } \\
\text { branches } \\
\text { /plant }\end{array}$ & $\begin{array}{l}\text { No. } \\
\text { leaves } \\
\text { /plant }\end{array}$ & $\begin{array}{c}\text { F.W. roots } \\
\text { /plant } \\
\text { (g) }\end{array}$ & $\begin{array}{c}\text { F.W. stem } \\
\text { /plant } \\
\text { (g) }\end{array}$ & $\begin{array}{c}\text { F.W. leaves } \\
\text { /plant } \\
\text { (g) }\end{array}$ & $\begin{array}{c}\text { Total F.W. } \\
\text { /plant } \\
\text { (g) }\end{array}$ \\
\hline & & \multicolumn{7}{|c|}{ First season (2014) } \\
\hline \multirow{6}{*}{ Bare plants } & Control & $62.00 \mathrm{de}$ & $9.500 \mathrm{~d}$ & $61.50 \mathrm{bcd}$ & $31.75 \mathrm{e}$ & $97.0 \mathrm{cde}$ & $281.8 \mathrm{fg}$ & $410.5 \mathrm{ef}$ \\
\hline & $\mathbf{C a}+\mathbf{B}$ & $67.50 \mathrm{bcd}$ & $9.500 \mathrm{~d}$ & $62.00 \mathrm{bcd}$ & $33.50 \mathrm{e}$ & $88.0 \mathrm{de}$ & 264.0fg & $385.5 \mathrm{ef}$ \\
\hline & $\mathbf{G A}_{3}$ & $62.00 \mathrm{de}$ & $11.00 \mathrm{~cd}$ & $59.50 \mathrm{~cd}$ & $43.50 \mathrm{e}$ & $97.5 \mathrm{cde}$ & $364.5 \mathrm{c}$ & $505.5 \mathrm{~d}$ \\
\hline & IAA & $61.00 \mathrm{de}$ & $9.500 \mathrm{~d}$ & $51.50 \mathrm{~d}$ & $34.50 \mathrm{e}$ & $79.7 \mathrm{e}$ & $235.2 \mathrm{~g}$ & $349.3 \mathrm{f}$ \\
\hline & $\mathbf{G A 3}+\mathbf{C a}+\mathbf{B}$ & $69.00 \mathrm{bcd}$ & $10.50 \mathrm{~cd}$ & $59.50 \mathrm{~cd}$ & $53.25 \mathrm{~d}$ & $112.0 \mathrm{~cd}$ & $348.5 \mathrm{~cd}$ & $513.8 \mathrm{~d}$ \\
\hline & $\mathbf{I A A}+\mathbf{C} \mathbf{a}+\mathbf{B}$ & $54.00 \mathrm{e}$ & $9.500 \mathrm{~d}$ & $50.00 \mathrm{~d}$ & $32.25 \mathrm{e}$ & $85.0 \mathrm{de}$ & 292.2ef & 409.3ef \\
\hline \multirow{6}{*}{$\begin{array}{l}\text { Floating cover } \\
\text { (Agryl) }\end{array}$} & Control & $76.50 \mathrm{~b}$ & $11.00 \mathrm{~cd}$ & $57.50 \mathrm{~cd}$ & $33.00 \mathrm{e}$ & $122.5 \mathrm{c}$ & 267.0fg & $422.5 \mathrm{e}$ \\
\hline & $\mathbf{C a}+\mathbf{B}$ & $86.00 \mathrm{a}$ & $13.50 \mathrm{bc}$ & $73.50 \mathrm{ab}$ & $51.50 \mathrm{~d}$ & $168.5 b$ & $463.0 \mathrm{ab}$ & $683.0 \mathrm{~b}$ \\
\hline & $\mathbf{G A}_{3}$ & $70.00 \mathrm{~cd}$ & $13.50 \mathrm{bc}$ & $66.00 \mathrm{bc}$ & $93.00 \mathrm{~b}$ & $180.5 b$ & $426.5 b$ & $700.0 \mathrm{~b}$ \\
\hline & IAA & $74.50 \mathrm{bc}$ & $17.00 \mathrm{a}$ & $80.50 \mathrm{a}$ & $117.00 \mathrm{a}$ & $209.0 \mathrm{a}$ & $507.5 \mathrm{a}$ & $833.5 \mathrm{a}$ \\
\hline & $\mathbf{G A}_{3}+\mathbf{C a}+\mathbf{B}$ & $65.00 \mathrm{~cd}$ & $11.50 \mathrm{~cd}$ & $70.50 \mathrm{abc}$ & $78.50 \mathrm{c}$ & $107.8 \mathrm{cde}$ & $302.5 \mathrm{def}$ & $488.8 \mathrm{~d}$ \\
\hline & $\mathbf{I A A}+\mathbf{C a}+\mathbf{B}$ & $70.00 \mathrm{bcd}$ & $15.00 \mathrm{ab}$ & $74.00 \mathrm{ab}$ & $84.50 \mathrm{bc}$ & $175.5 b$ & 338.5 cde & $598.5 \mathrm{c}$ \\
\hline
\end{tabular}

Second season (2015)

\begin{tabular}{lcccccccc}
\hline & Control & $58.67 \mathrm{~h}$ & $10.00 \mathrm{bc}$ & $58.00 \mathrm{de}$ & $33.30 \mathrm{f}$ & $88.6 \mathrm{~h}$ & $261.0 \mathrm{f}$ & $383.0 \mathrm{~cd}$ \\
\multirow{5}{*}{ Bare plants } & $\mathbf{C a}+\mathbf{B}$ & $64.33 \mathrm{fg}$ & $8.700 \mathrm{c}$ & $61.00 \mathrm{~cd}$ & $39.00 \mathrm{e}$ & $93.0 \mathrm{gh}$ & $275.5 \mathrm{e}$ & $407.5 \mathrm{~cd}$ \\
& $\mathbf{G A}_{3}$ & $63.00 \mathrm{fg}$ & $9.733 \mathrm{bc}$ & $57.00 \mathrm{de}$ & $38.66 \mathrm{e}$ & $94.6 \mathrm{~g}$ & $256.5 \mathrm{f}$ & $389.9 \mathrm{~cd}$ \\
& $\mathbf{I A A}$ & $64.67 \mathrm{f}$ & $8.800 \mathrm{c}$ & $53.67 \mathrm{e}$ & $39.20 \mathrm{e}$ & $80.6 \mathrm{i}$ & $243.0 \mathrm{~g}$ & $362.9 \mathrm{~d}$ \\
& $\mathbf{G A}_{\mathbf{3}}+\mathbf{C a}+\mathbf{B}$ & $72.33 \mathrm{~cd}$ & $9.400 \mathrm{c}$ & $65.33 \mathrm{bc}$ & $42.00 \mathrm{e}$ & $103.0 \mathrm{f}$ & $292.5 \mathrm{~d}$ & $437.5 \mathrm{bc}$ \\
& $\mathbf{I A A}+\mathbf{C a}+\mathbf{B}$ & $62.33 \mathrm{~g}$ & $9.200 \mathrm{c}$ & $61.00 \mathrm{~cd}$ & $40.50 \mathrm{f}$ & $91.0 \mathrm{gh}$ & $279.0 \mathrm{e}$ & $410.5 \mathrm{c}$ \\
& & & & & & & & \\
Floating cover & $\mathbf{C o n t r o l}$ & $68.67 \mathrm{e}$ & $10.70 \mathrm{abc}$ & $54.67 \mathrm{e}$ & $33.50 \mathrm{f}$ & $113.7 \mathrm{e}$ & $247.5 \mathrm{~g}$ & $394.7 \mathrm{~cd}$ \\
(Agryl) & $\mathbf{C a}+\mathbf{B}$ & $83.00 \mathrm{a}$ & $11.30 \mathrm{bc}$ & $68.67 \mathrm{ab}$ & $41.30 \mathrm{e}$ & $144.3 \mathrm{c}$ & $310.5 \mathrm{c}$ & $496.1 \mathrm{~b}$ \\
& $\mathbf{G A}$ & $73.33 \mathrm{bc}$ & $11.70 \mathrm{abc}$ & $69.00 \mathrm{ab}$ & $63.20 \mathrm{~d}$ & $151.0 \mathrm{~b}$ & $315.3 \mathrm{c}$ & $529.5 \mathrm{~b}$ \\
& $\mathbf{I A A}$ & $74.67 \mathrm{~b}$ & $13.10 \mathrm{ab}$ & $74.67 \mathrm{a}$ & $78.50 \mathrm{c}$ & $162.7 \mathrm{a}$ & $337.5 \mathrm{a}$ & $578.7 \mathrm{a}$ \\
& $\mathbf{G A}_{\mathbf{3}}+\mathbf{C a}+\mathbf{B}$ & $71.00 \mathrm{~d}$ & $13.70 \mathrm{a}$ & $72.33 \mathrm{a}$ & $85.10 \mathrm{~b}$ & $148.3 \mathrm{bc}$ & $326.3 \mathrm{~b}$ & $559.7 \mathrm{ab}$ \\
& $\mathbf{I A A}+\mathbf{C a}+\mathbf{B}$ & $72.00 \mathrm{~cd}$ & $12.80 \mathrm{ab}$ & $69.00 \mathrm{ab}$ & $91.00 \mathrm{a}$ & $136.7 \mathrm{~d}$ & $342.1 \mathrm{a}$ & $569.8 \mathrm{ab}$ \\
\hline
\end{tabular}

Values having the same alphabetical letter (s) did not significantly differ at 0.05 level of significance according to Duncan's multiple range test 
Table (6): Effect of floating cover (Agryl) and fruit setting substances on dry weight of tomato plants

\section{Dry weight / plant (g)}

Treatments
D.W. roots
D.W. stem
D.W. leaves

Total dry weight

First season (2014)

\begin{tabular}{|c|c|c|c|c|}
\hline Bare plants & $11.79 b$ & $22.12 b$ & $49.83 b$ & $83.76 b$ \\
\hline $\begin{array}{l}\text { Floating cover } \\
\text { (Agryl) }\end{array}$ & $18.87 \mathrm{a}$ & $33.56 \mathrm{a}$ & $73.45 \mathrm{a}$ & $125.83 a$ \\
\hline
\end{tabular}

Second season (2015)

\begin{tabular}{|c|c|c|c|c|}
\hline Bare plants & $13.01 \mathrm{a}$ & $21.91 b$ & $45.83 b$ & $80.78 b$ \\
\hline $\begin{array}{l}\text { Floating cover } \\
\text { (Agryl) }\end{array}$ & $15.56 \mathrm{a}$ & $32.27 \mathrm{a}$ & $68.92 \mathrm{a}$ & $116.76 \mathrm{a}$ \\
\hline
\end{tabular}

(Agryl)

First season (2014)

\begin{tabular}{|c|c|c|c|c|}
\hline Control & $10.75 c$ & $21.88 \mathrm{c}$ & $45.13 b$ & $77.77 \mathrm{c}$ \\
\hline $\mathbf{C a}+\mathbf{B}$ & $9.50 \mathrm{c}$ & $23.88 b c$ & $63.88 \mathrm{a}$ & $97.25 b$ \\
\hline $\mathbf{G A}_{3}$ & $13.75 b c$ & $27.75 b$ & $67.63 a$ & $109.10 \mathrm{~b}$ \\
\hline IAA & $26.63 a$ & $38.13 \mathrm{a}$ & $66.88 \mathrm{a}$ & $131.63 a$ \\
\hline $\mathbf{G A}_{3}+\mathbf{C a}+\mathbf{B}$ & $16.13 b$ & $26.81 b c$ & $63.25 \mathrm{a}$ & $106.20 \mathrm{~b}$ \\
\hline $\mathbf{I A A}+\mathbf{C a}+\mathbf{B}$ & $15.25 b$ & $28.63 b$ & $63.13 \mathrm{a}$ & $107.01 \mathrm{~b}$ \\
\hline
\end{tabular}

\begin{tabular}{|c|c|c|c|c|}
\hline \multicolumn{5}{|c|}{ Second season (2015) } \\
\hline Control & $10.85 \mathrm{e}$ & $20.25 \mathrm{e}$ & $39.07 \mathrm{~b}$ & $70.20 \mathrm{c}$ \\
\hline $\mathbf{C a}+\mathbf{B}$ & $12.15 \mathrm{de}$ & $24.33 d$ & $59.43 a$ & $95.92 b$ \\
\hline $\mathbf{G A}_{3}$ & $13.48 \mathrm{~cd}$ & $26.00 \mathrm{c}$ & $59.30 \mathrm{a}$ & $98.80 \mathrm{~b}$ \\
\hline IAA & $14.50 \mathrm{c}$ & $31.50 \mathrm{a}$ & $61.55 \mathrm{a}$ & $107.60 \mathrm{a}$ \\
\hline $\mathbf{G A}_{3}+\mathbf{C a}+\mathbf{B}$ & $16.08 b$ & $32.67 \mathrm{a}$ & $62.65 \mathrm{a}$ & $111.40 \mathrm{a}$ \\
\hline $\mathbf{I A A}+\mathbf{C a}+\mathbf{B}$ & $18.65 \mathrm{a}$ & $27.83 b$ & $62.30 \mathrm{a}$ & $108.80 \mathrm{a}$ \\
\hline
\end{tabular}

Values having the same alphabetical letter (s) did not significantly differ at 0.05 level of significance according to Duncan's multiple range test

\section{Effect of interaction between floating covers and fruit setting substances}

Data in Table (7) show significant differences among the treatments (the interaction between float covering and fruit setting substances) on dry weight of tomato plant. The data illustrated that spraying tomato plants grown under spunbonded with IAA was the superior treatment in both seasons, where dry weight of roots ( $1^{\text {st }}$ season), dry weight of stem, leaves and consequently total dry weight of plants were increased (in both seasons).

It could be notice that plants grown under Agryl recorded high values of plant height, number of both branches and leaves/plant, total fresh and dry weight/plant in spite of exposing the plants to high temperature as shown in Table (3). That may be owe to the engendered substances such as proline or unique set of heat shock protein under high temperature which help plants to resist the heat stress (Ördog, 2011). Proline could increase chickpea plant growth under high temperature $\left(40 / 35^{\circ} \mathrm{c}\right.$ and $40 / 45^{\circ} \mathrm{c}$ day/night via reducing the cellular injury and protection of some vital enzymes related to carbon and oxidative metabolism (Kaushal et al., 2011). In addition, proline considers the precursor of pigment and reduces the undesirable effect of free radicals. Proline plays roles as osmoregulation maintenancing membrane protein stability; (Hare et al., 2003). Also, growth of beetroot increased with proline as recorded by El-Sherbeny and Da Silva (2013). 
Table (7): Effect of interaction between floating cover (Agryl) and fruit setting substances on dry weight of tomato plants

\begin{tabular}{|c|c|c|c|c|c|}
\hline \multirow{2}{*}{ Treatments } & & \multicolumn{4}{|c|}{ Dry weight / plant (gm) } \\
\hline & & D.W. roots & D.W. stem & \multirow[t]{2}{*}{ D.W. leaves } & \multirow[t]{2}{*}{ Total dry weight } \\
\hline & & First $s$ & (2014) & & \\
\hline \multirow{6}{*}{ Bare plants } & Control & $12.50 \mathrm{~cd}$ & $23.00 \mathrm{def}$ & $44.75 \mathrm{e}$ & $80.27 \mathrm{ef}$ \\
\hline & $\mathbf{C a}+\mathbf{B}$ & $10.00 \mathrm{~cd}$ & $20.50 \mathrm{efg}$ & $46.00 \mathrm{e}$ & $76.50 \mathrm{f}$ \\
\hline & $\mathbf{G A}_{3}$ & $12.00 \mathrm{~cd}$ & $25.25 \mathrm{cde}$ & $58.00 \mathrm{~d}$ & $95.27 \mathrm{de}$ \\
\hline & IAA & $10.75 \mathrm{~cd}$ & $19.75 \mathrm{fg}$ & $43.25 \mathrm{e}$ & $73.77 \mathrm{f}$ \\
\hline & $\mathbf{G A}_{3}+\mathbf{C a}+\mathbf{B}$ & $13.75 \mathrm{bcd}$ & $27.75 \mathrm{~cd}$ & $57.50 \mathrm{~d}$ & $99.00 \mathrm{~cd}$ \\
\hline & $\mathbf{I A A}+\mathbf{C a}+\mathbf{B}$ & $11.75 \mathrm{~cd}$ & $16.50 \mathrm{~g}$ & $49.50 \mathrm{de}$ & $77.77 f$ \\
\hline \multirow{6}{*}{$\begin{array}{l}\text { Floating cover } \\
\text { (Agryl) }\end{array}$} & Control & $9.00 \mathrm{~d}$ & $20.75 \mathrm{efg}$ & $45.50 \mathrm{e}$ & $75.27 f$ \\
\hline & $\mathrm{Ca}+\mathrm{B}$ & $9.00 \mathrm{~d}$ & $27.25 \mathrm{~cd}$ & $81.75 \mathrm{ab}$ & $118.00 \mathrm{~b}$ \\
\hline & $\mathbf{G A}_{3}$ & $15.50 \mathrm{bc}$ & $30.25 \mathrm{c}$ & $77.25 \mathrm{bc}$ & $123.00 \mathrm{~b}$ \\
\hline & IAA & $42.5 \mathrm{a}$ & $56.50 \mathrm{a}$ & $90.50 \mathrm{a}$ & $189.5 \mathrm{a}$ \\
\hline & $\mathbf{G A}_{3}+\mathbf{C a}+\mathbf{B}$ & $18.50 \mathrm{~b}$ & $25.87 \mathrm{cde}$ & $69.00 \mathrm{c}$ & $113.40 \mathrm{bc}$ \\
\hline & $\mathbf{I A A}+\mathbf{C a}+\mathbf{B}$ & $18.75 b$ & $40.75 b$ & $76.75 b c$ & $136.25 b$ \\
\hline \multicolumn{6}{|c|}{ Second season (2015) } \\
\hline \multirow{6}{*}{ Bare plants } & Control & $11.00 \mathrm{fg}$ & $21.00 \mathrm{~g}$ & $39.63 f$ & $71.70 \mathrm{f}$ \\
\hline & $\mathbf{C a}+\mathbf{B}$ & $13.00 \mathrm{de}$ & $23.33 \mathrm{f}$ & $47.80 \mathrm{de}$ & $84.13 \mathrm{e}$ \\
\hline & GA3 & $12.75 \mathrm{def}$ & $24.50 \mathrm{ef}$ & $46.30 \mathrm{e}$ & $83.60 \mathrm{e}$ \\
\hline & IAA & $13.00 \mathrm{de}$ & $18.33 \mathrm{~h}$ & $41.50 \mathrm{f}$ & $72.83 \mathrm{f}$ \\
\hline & $\mathbf{G A}_{3}+\mathbf{C a}+\mathbf{B}$ & $14.50 \mathrm{~cd}$ & $26.00 \mathrm{de}$ & $51.00 \mathrm{~d}$ & $91.50 \mathrm{~d}$ \\
\hline & $\mathbf{I A A}+\mathbf{C a}+\mathbf{B}$ & $13.80 \mathrm{~d}$ & $18.33 \mathrm{~h}$ & $48.80 \mathrm{de}$ & $80.93 \mathrm{e}$ \\
\hline \multirow{6}{*}{$\begin{array}{l}\text { Floating cover } \\
\text { (Agryl) }\end{array}$} & Control & $10.70 \mathrm{~g}$ & $19.50 \mathrm{gh}$ & $38.50 \mathrm{f}$ & $68.70 f$ \\
\hline & $\mathbf{C a}+\mathbf{B}$ & $11.30 \mathrm{efg}$ & $25.33 \mathrm{e}$ & $71.07 \mathrm{c}$ & $107.70 \mathrm{c}$ \\
\hline & $\mathbf{G A}_{3}$ & $14.20 \mathrm{~d}$ & $27.50 \mathrm{~d}$ & $72.30 \mathrm{bc}$ & $114.00 \mathrm{c}$ \\
\hline & IAA & $16.00 \mathrm{bc}$ & $44.67 \mathrm{a}$ & $81.60 \mathrm{a}$ & $142.30 \mathrm{a}$ \\
\hline & $\mathbf{G A}_{3}+\mathbf{C a}+\mathbf{B}$ & $17.67 \mathrm{~b}$ & $39.33 b$ & $74.30 \mathrm{bc}$ & $131.30 \mathrm{~b}$ \\
\hline & $\mathbf{I A A}+\mathbf{C a}+\mathbf{B}$ & $23.50 \mathrm{a}$ & $37.33 c$ & $75.80 \mathrm{~b}$ & $136.60 \mathrm{ab}$ \\
\hline
\end{tabular}

Values having the same alphabetical letter (s) did not significantly differ at 0.05 level of significance according to Duncan's multiple range test

\section{Proline concentration}

\section{Effect of floating covers}

It is obvious from the data in Fig. (1) that covering plants with Agryl increased the content of proline in tomato leaves compared to uncovered (bare) plants in both seasons. High concentration of proline under Agryl covers considered as a react to resist the abnormal conditions as temperature. Székely et al. (2008) and Verburggen and Hermans (2008) reported an accumulation of proline under various osmotic stress. Maggio et al. (2002) observed an accumulation of proline in cells grown under normal or in mild hyper osmotic stress, and the highest levels were observed in pollen grains and seeds while the lowest values were in roots. In this direction, content of proline was increased in chickpea leaves exposed to high temperature 40/35c ${ }^{\circ}$ for day/night; (Kaushal et al., 2011).

\section{Effect of fruit setting substances}

Data in Fig. (2) reveal the effect of fruit setting substances on proline content in tomato leaves grown under high temperature conditions. It was clear that spraying with IAA or $\mathrm{GA}_{3}$ was the best treatment in the $1^{\text {st }}$ and $2^{\text {nd }}$ seasons, respectively, followed by spraying with $\mathrm{GA}_{3}+\mathrm{Ca}+\mathrm{B}$ and IAA $+\mathrm{Ca}+\mathrm{B}$ in both seasons.

\section{Effect of interaction between floating covers and fruit setting substance}

Data in Fig. (3) show the effect of interaction between covering or without covering plants with Agryl and spraying with fruit setting substances on tomato 
leave proline contents. The data showed that spraying tomato plants grown under floating cover with $\mathrm{GA}_{3}$, $\mathrm{IAA}, \mathrm{GA}_{3}+\mathrm{Ca}+\mathrm{B}$ and $\mathrm{IAA}+\mathrm{Ca}+\mathrm{B}$ recorded the highest proline concentration in tomato leaves. On the other side, the lowest concentrations of proline were recorded with all fruit setting substances under bare plants. The obtained results were similarly in both seasons. These results might be owe to the effect of floating covers which increased the air temperature as shown in Table (3) which induced a heat stress on tomato plants.

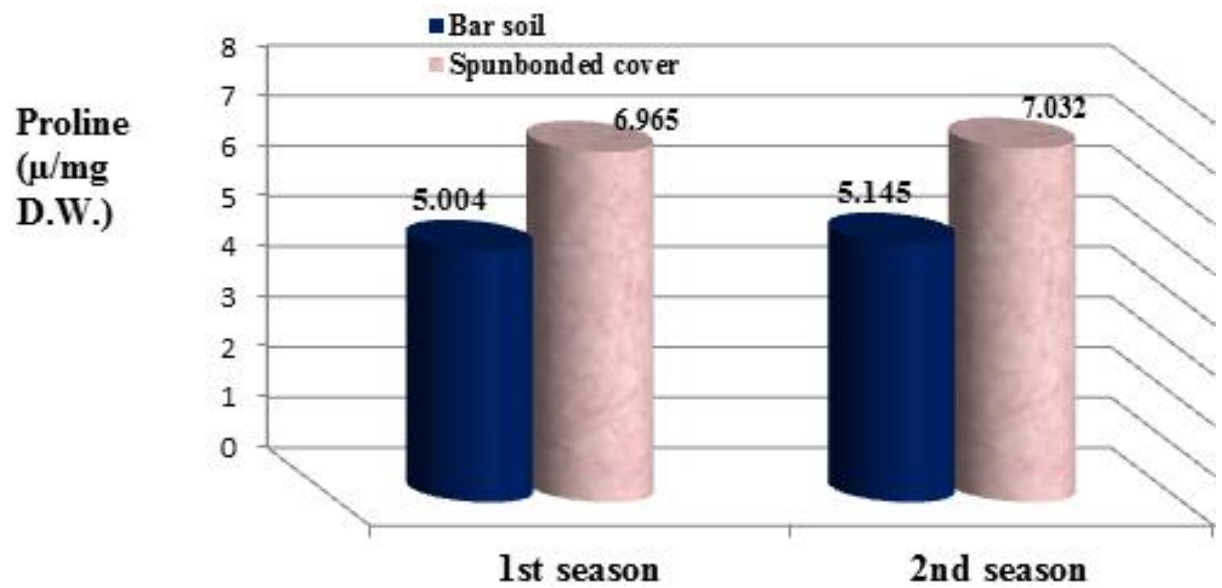

Fig. (1): Effect of application of spunbonded (Agryl) cover on proline concentration in tomato leaves in 2014 and 2015 seasons

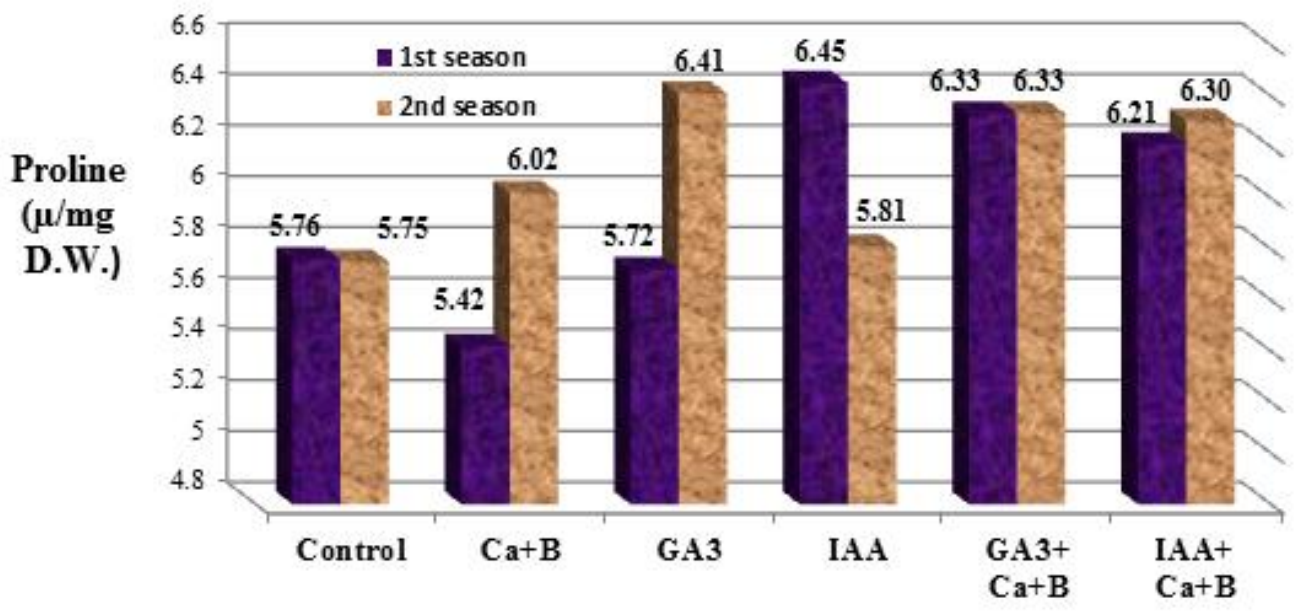

Fig. (2): Effect of fruit setting substances on proline concentration in tomato leaves in 2014 and 2015 seasons

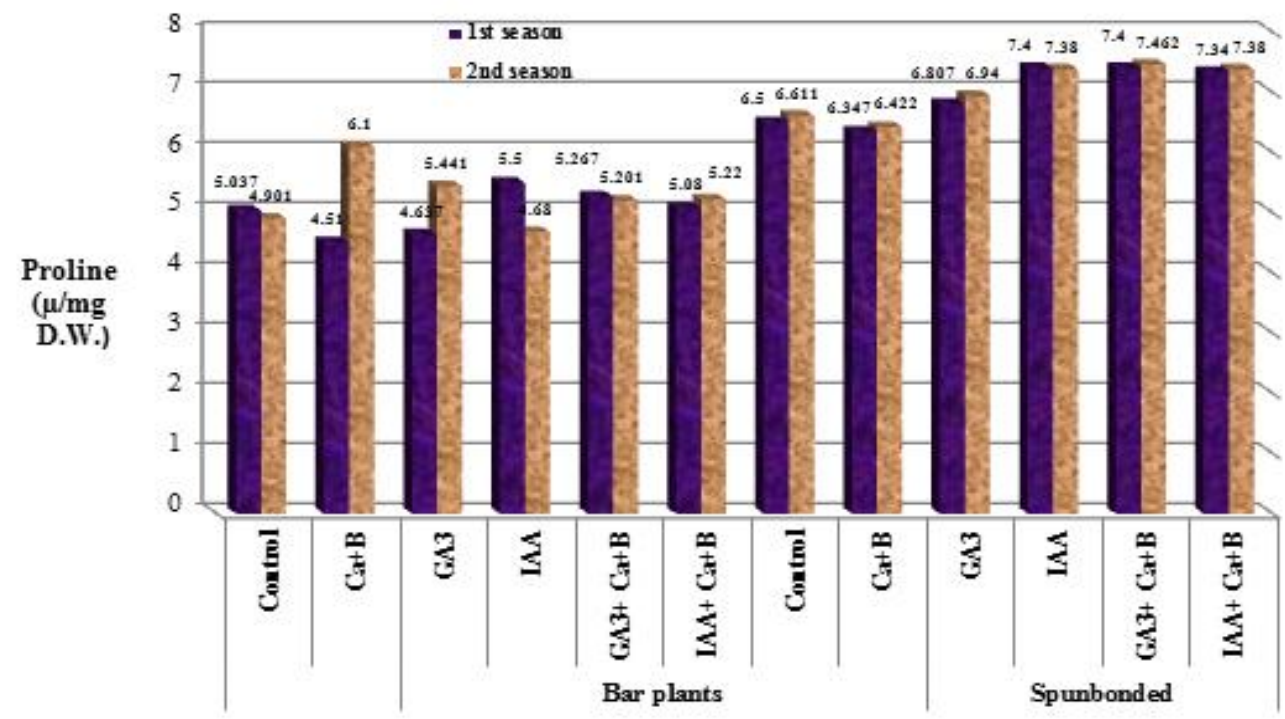

Fig. (3): Effect of interaction between spunbnded covers (Agryl) and fruit setting substances on proline concentration in tomato leaves in 2014and 2015 seasons 


\section{Total chlorophyll}

\section{Effect of floating covers}

Data in Fig. (4) show significant effect due to applied or unapplied floating cover sheets on total chlorophyll content in tomato leaves grown under high temperature seasons. The data illustrated that total chlorophyll content in tomato leaves increased in bare plants compared to covering plants with Agryl. Despite the decrease in chlorophyll content in plants under Agryl compared to the bare plants, but this content was not far from bare plants. This may be due to the increments in proline contents as shown in Fig.1 under Agryl covers. Increasing in endogenous proline due to high temperature enhanced chlorophyll content and this may be due to its role in stability of sub cellular structure like chloroplast and their membrane as reported by (Aggarwal et al., 2011). Kaushal et al. (2011) found a reduction in chickpea chlorophyll content under high temperature. In the same line it was reported that heat stress reduced the chlorophyll content and photosynthetic active radiation (Zhou et al., 2016).
Application of floating row covers decreased the photo synthetic active radiation (Saidi et al., 2013).

\section{Effect of fruit setting substances}

Data presented in Fig. (5) illustrate that all fruit setting substances applied as foliar spray increased the content of total chlorophyll in tomato leaves compared to control treatment. Spraying with IAA followed by spraying with $\mathrm{IAA}+\mathrm{Ca}+\mathrm{B}$ were the best treatments in both seasons.

\section{Effect of interaction between floating covers and fruit setting substances}

Data in Fig. (6) reveal that all the interaction treatments between bare plants and fruit setting substances increased the content of total chlorophyll compared to the same treatments under covering with Agryl.

It was obvious from the same Figure that spraying bare tomato plants by $\mathrm{IAA}+\mathrm{Ca}+\mathrm{B}$ was the best interaction treatment followed by spraying with IAA.

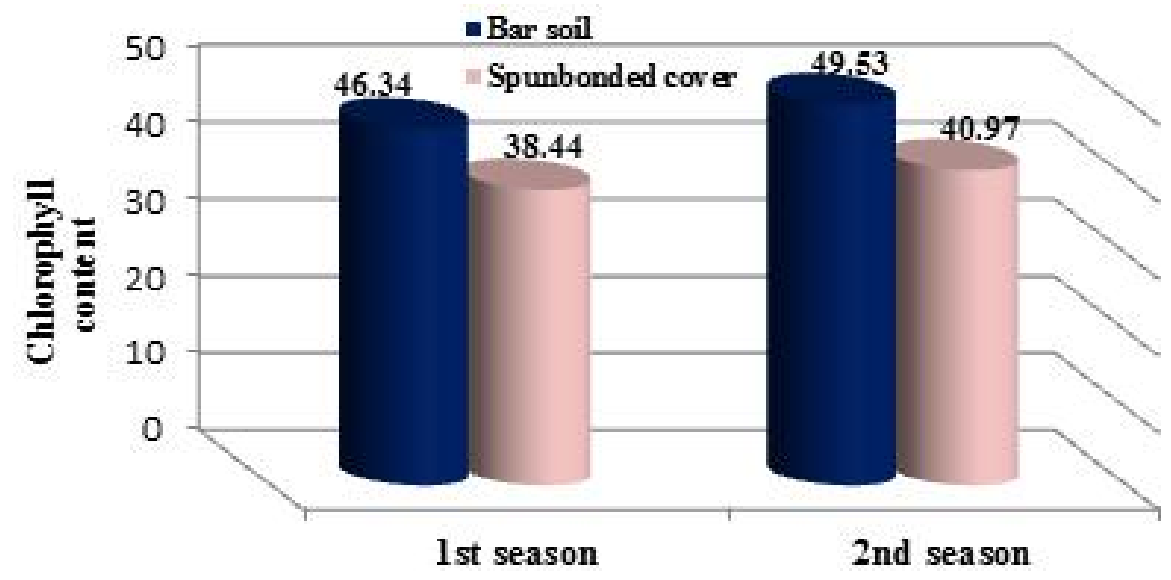

Fig. (4): Effect of application of spunbonded cover (Agryl) on total chlorophyll content in tomato leaves measured by digital chlorophyll meter in 2014 and 2015 seasons

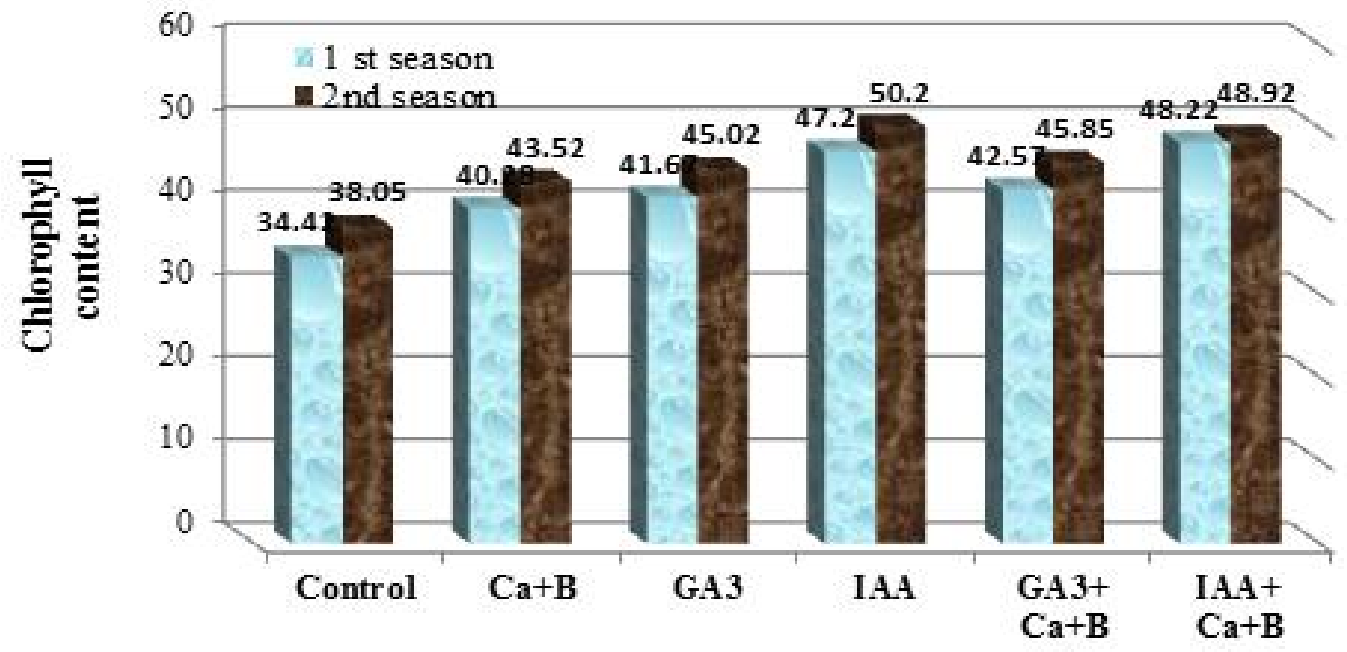

Fig. (5): Effect of fruit setting substances on chlorophyll content in tomato leaves in 2014 and 2015 seasons 


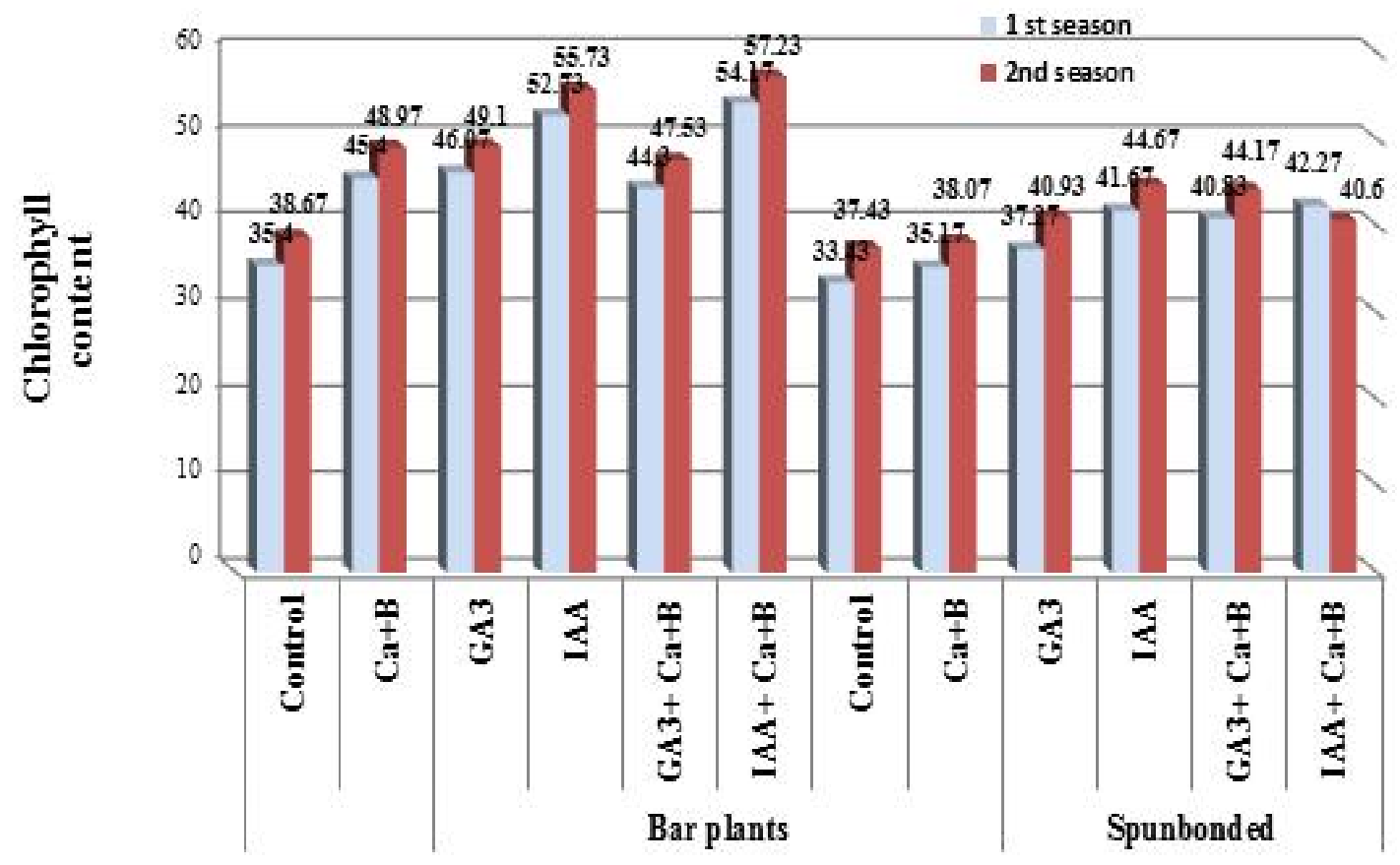

Fig. (6): Effect of interaction between spunbonded covers (Agryl) and fruit setting substances on chlorophyll content in tomato leaves in 2014 and 2015 seasons

\section{Fruit setting (\%)}

\section{Effect of floating covers}

Data in Table (8) show that there was no significant effect on $1^{\text {st }}$ cluster fruit setting $\%$ in both seasons as well as the fruit setting (\%) of the third cluster in the $1^{\text {st }}$ season, while it increased with application of floating cover in the $2^{\text {nd }}$ season. The fruit setting (\%) of the $2^{\text {nd }}$ cluster was increased with tomato plants covered by Agryl compared to the bare plants in both seasons. The increment in fruit setting (\%) of the $2^{\text {nd }}$ cluster and the $3^{\text {rd }}$ cluster in the $2^{\text {nd }}$ one caused increments in the average fruit setting \% of the three clusters. The increased average fruit setting (\%) due to application of Agryl compared to bare plants may be attributed to the increment in plant growth, fresh weight and dry weight of plant (Tables 4 and 6).

Table (8): Effect of floating cover (Agryl) and fruit setting substances on tomato fruit setting (\%)

\begin{tabular}{|c|c|c|c|c|c|c|c|c|}
\hline \multirow[b]{2}{*}{ Treatments } & \multicolumn{8}{|c|}{ Fruit setting (\%) } \\
\hline & $\begin{array}{c}1^{\text {st }} \\
\text { cluster }\end{array}$ & $\begin{array}{c}2^{\text {nd }} \\
\text { cluster }\end{array}$ & $\begin{array}{c}3^{\text {rd }} \\
\text { cluster }\end{array}$ & $\begin{array}{c}\text { Avg. } \\
1^{\text {st }}+2^{\text {nd }}+3^{\text {rd }} \\
\text { clusters }\end{array}$ & $\begin{array}{c}1^{\text {st }} \\
\text { cluster }\end{array}$ & $2^{\text {nd }}$ cluster & $3^{\text {rd }}$ cluster & $\begin{array}{c}\text { Avg. } \\
1^{\text {st }}+2^{\text {nd }}+3^{\text {rd }} \\
\text { clusters }\end{array}$ \\
\hline & \multicolumn{4}{|c|}{ First season (2014) } & \multicolumn{4}{|c|}{ Second season (2015) } \\
\hline Bare plants & $77.08 \mathrm{a}$ & $71.75 b$ & $72.08 \mathrm{a}$ & $73.54 b$ & $79.37 \mathrm{a}$ & $71.79 b$ & $69.65 b$ & $73.60 \mathrm{~b}$ \\
\hline $\begin{array}{l}\text { Floating cover } \\
\text { (Agryl) }\end{array}$ & $77.43 \mathrm{a}$ & $84.29 \mathrm{a}$ & $75.08 \mathrm{a}$ & $78.93 a$ & $76.57 \mathrm{a}$ & $84.52 a$ & $75.70 \mathrm{a}$ & $78.93 a$ \\
\hline Control & $39.17 \mathrm{c}$ & $68.50 \mathrm{~b}$ & $45.83 b$ & $50.89 b$ & $40.42 d$ & $70.75 c$ & $47.50 \mathrm{c}$ & $52.89 \mathrm{c}$ \\
\hline $\mathbf{C a}+\mathbf{B}$ & $71.25 \mathrm{~b}$ & $68.75 b$ & $45.42 b$ & $61.81 b$ & $73.13 c$ & $68.13 c$ & $41.46 \mathrm{c}$ & $60.90 \mathrm{~b}$ \\
\hline $\mathbf{G A}_{3}$ & $100.00 \mathrm{a}$ & $74.00 \mathrm{~b}$ & $95.00 \mathrm{a}$ & $89.67 \mathrm{a}$ & $100.00 \mathrm{a}$ & $73.50 \mathrm{bc}$ & $92.50 \mathrm{a}$ & $88.67 \mathrm{a}$ \\
\hline IAA & $85.00 \mathrm{ab}$ & $74.38 b$ & $83.25 \mathrm{a}$ & $80.88 \mathrm{a}$ & $87.50 \mathrm{~b}$ & $71.56 \mathrm{c}$ & $91.63 \mathrm{a}$ & $83.56 \mathrm{a}$ \\
\hline $\mathbf{G} \mathbf{A}_{3}+\mathbf{C a}+\mathbf{B}$ & $92.72 \mathrm{a}$ & $82.50 \mathrm{ab}$ & $89.50 \mathrm{a}$ & $88.24 \mathrm{a}$ & $89.09 \mathrm{~b}$ & $85.00 \mathrm{~b}$ & $84.25 \mathrm{ab}$ & $86.11 \mathrm{a}$ \\
\hline $\mathbf{I A A}+\mathbf{C a}+\mathbf{B}$ & $75.42 b$ & $100.00 \mathrm{a}$ & $82.50 \mathrm{a}$ & $85.97 \mathrm{a}$ & $77.71 \mathrm{c}$ & $100.00 \mathrm{a}$ & $78.75 b$ & $85.49 a$ \\
\hline
\end{tabular}




\section{Effect of fruit setting substances}

Data in Table (8) illustrate significant effects for application of fruit setting substances on tomato fruit setting (\%). The data revealed that spraying tomato with $\mathrm{GA}_{3}$ was the best treatment for fruit setting (\%) in the $1^{\text {st }}$ cluster in both seasons followed by spraying with $\mathrm{GA}_{3}+\mathrm{Ca}+\mathrm{B}$ and IAA in the $1^{\text {st }}$ season, while the highest fruit setting $\%$ in $2^{\text {nd }}$ cluster was recorded with application of $\mathrm{IAA}+\mathrm{Ca}+\mathrm{B}$ in both seasons. In addition, the highest fruit setting $\%$ in the $3^{\text {rd }}$ cluster was achieved with spraying $\mathrm{GA}_{3}$ and IAA in both seasons as well as spraying with $\mathrm{GA}_{3}+\mathrm{Ca}+\mathrm{B}$ or $\mathrm{IAA}+\mathrm{Ca}+\mathrm{B}$ in the $1^{\text {st }}$ one. The same previous four treatments $\left(\mathrm{GA}_{3}, \mathrm{IAA}\right.$, $\mathrm{GA}_{3}+\mathrm{Ca}+\mathrm{B}$ and $\left.\mathrm{IAA}+\mathrm{Ca}+\mathrm{B}\right)$ increased the average fruit setting ( $\%$ ) of the $2^{\text {nd }}$ cluster. It was obvious that the increment in $3^{\text {rd }}$ cluster fruit setting due to spraying with $\mathrm{GA}_{3}$, IAA, $\mathrm{GA}_{3}+\mathrm{Ca}+\mathrm{B}$ and $\mathrm{IAA}+\mathrm{Ca}+\mathrm{B}$ might be attribute to the increment in $3^{\text {rd }}$ cluster fruit setting. Finally, it could be concluded that spraying with $\mathrm{GA}_{3}$ was the superior treatment for average fruit setting (\%) followed by spraying with $\mathrm{GA}_{3}+\mathrm{Ca}+\mathrm{B}, \mathrm{IAA}+\mathrm{Ca}+\mathrm{B}$ and IAA.

\section{Effect of interaction between floating covers and fruit setting substances}

Data in Table (9) reveal significant effect of interaction between covering tomato plants with Agryl and enhancing fruit setting substances on fruit setting (\%). The data illustrated that the interaction between application of Agryl with spraying with $\mathrm{GA}_{3}$, $\mathrm{IAA}+\mathrm{Ca}+\mathrm{B}$ as well as $\mathrm{GA}_{3}+\mathrm{Ca}+\mathrm{B}$, or spraying with IAA enhanced the fruit setting (\%) in both seasons, and bare plants with $\mathrm{GA}_{3}$ and $\mathrm{GA}_{3}+\mathrm{Ca}+\mathrm{B}$ in the $1^{\text {st }}$ season. In this connection, many researchers found decreases in pollen viability and fruit set (\%) with rising in temperature (Peet et al., 1997; Zhou et al., 2016). However the obtained results showed increment in fruit setting under covering plants with Agryl and this might be due to the vital roles of IAA, $\mathrm{GA}_{3}$ and the synergistic effect by the interaction of $\mathrm{Ca}+\mathrm{B}$. IAA plays a fundamental role in successful pollination and helps ovary to grow rapidly without pollination and fertilization. Additionally, $\mathrm{GA}_{3}$ can develop pollen grain and pollen tube as well as the ovary auxin content to the level which trigger fruit to grow (Smit and Combrink 2005; de Jong et al., 2009; Desouky et al., 2009; Haque et al., 2011; Ördog, 2011; Rab and Haq, 2012; Pattison et al., 2014).

\section{Yield and its components}

\section{Effect of floating covers}

Data presented in Table (10) show distinctly the effect for application or without application of floating covers on marketable, unmarketable and total yield of tomato plant. The data show that bare plants increased yield/plant, yield/fed., total yield, unmarketable yield as well as number of seeds/fruit. The yield and its components were increased in spite of slight increment in fruit setting (\%) by application of Agryl, that may be owe to the increment in fruit weight of bare plants which increased the yield of plant and consequently the yield/fed. The increment in number of seeds was taken as indicator to developing the size of fruits and the peak of auxin in fruits (Pattison et al. 2014) where seeds are considered the source of auxin in fruit and it promotes fruit growth by cell division and cell expansion. Peet et al. (1997) found a reduction in number of seeds/fruit due to rising the daily average of temperature from 25 to $26^{\circ} \mathrm{C}$ and from 28 to $29^{\circ} \mathrm{C}$. The decline in number of seeds/ plant under high temperature may be owed to the decrease in pollen viability and the inhibition of pollen tube growth and fertility (Leah and Aloni, 2002; Kafizadeh et al., 2008).

\section{Effect of fruit setting substances}

It was clear from the data presented in Table (10) that the application of $\mathrm{IAA}+\mathrm{Ca}+\mathrm{B}$ increased significantly the yield of tomato plant, marketable yield (ton/fed.) and hence total yield /fed. as well as number of seeds/fruit. The application of IAA separately increased the unmarketable yield/fed. (ton/fed.) measured as fruits infected by blossom-end rot. The obtained results were true in both seasons. In addition application of $\mathrm{Ca}+\mathrm{B}$ or $\mathrm{GA}_{3}+\mathrm{Ca}+\mathrm{B}$ recorded the second rank of marketable and total yield (ton/fed.).The increments in marketable yield (ton/fed.)were $35.86 \%$ and $39.30 \%$ over the control treatment followed by spraying with $\mathrm{GA}_{3}+\mathrm{Ca}+\mathrm{B}$ which recorded increments by $25.42 \%$ and $30.50 \%$, while it increased by $24.66 \%$ and $31.62 \%$ with $\mathrm{Ca}+\mathrm{B}$ in the $1^{\text {st }}$ and $2^{\text {nd }}$ seasons, respectively. The increase of yield may partially due to the high content of chlorophyll pigment (Fig. 4) leading to high photo-assimilation and consequently increasing in total fresh and dry weight of plant in addition to increasing in fruit setting (\%) and so, increase in yield per plant and per feddan as shown in Tables (4, 6 and $8)$.

\section{Effect of interaction between floating covers and fruit setting substances}

Data presented in Table (11) reveal that spraying bare plant with $\mathrm{IAA}+\mathrm{Ca}+\mathrm{B}$ or $\mathrm{GA}_{3}+\mathrm{Ca}+\mathrm{B}$ were the superior treatments in the $1^{\text {st }}$ season respectively where they increased the marketable yield of plant and per feddan, total yield /feddan, and number of seeds/fruit in both seasons. The relative increase in marketable yield /fed. was $39.48 \%, 38.86 \%$ and $35.77 \%$ for the same abovementioned treatments over the control treatments of bare plants in the $1^{\text {st }}$ season, respectively, while spraying with IAA $+\mathrm{Ca}+\mathrm{B}$ was the best treatment under application of floating cover which increased the marketable yield/fed. by $31.62 \%$ over the control of covered plants. In the $2^{\text {nd }}$ season, spraying plants with $\mathrm{IAA}+\mathrm{Ca}+\mathrm{B}$ or $\mathrm{GA}_{3}+\mathrm{Ca}+\mathrm{B}$ increased the yield /plant, marketable yield/fed., and the increment in relative yield were $47.75 \%$ and $45.50 \%$ for the previous treatment, respectively, compared to the control of bare plants. On the other hand, application of IAA alone increased the unmarketable yield (ton/fed.). It could be concluded that spraying tomato plants with $\mathrm{IAA}+\mathrm{Ca}+\mathrm{B}$ or $\mathrm{GA}_{3}+\mathrm{Ca}+\mathrm{B}$ were the superior treatments for the marketable and total yield (ton/fed.) for bare plants in both seasons. The increments in yield /plant caused an increment in marketable yield/ fed. The increase in bare plant yield may be owe to the increase in fruit weight. Fruit weight was decreased under high temperature that 
may be owe to the fast developing and fruits harvested rapidly before reaching the full size (Peet et al., 1997). The increments in yield with application of IAA or $\mathrm{GA}_{3}$ with $\mathrm{Ca}+\mathrm{B}$ may be owe to their effects on fruit developing, cells division and expansion which result in increasing fruit size and consequently increasing yield/plant and per fed.

Table (9): Effect of interaction between floating cover (Agryl) and fruit setting substances on tomato fruit setting (\%)

\begin{tabular}{|c|c|c|c|c|c|c|c|c|c|}
\hline \multirow{3}{*}{ Treatments } & & \multicolumn{8}{|c|}{ Fruit setting (\%) } \\
\hline & & $\begin{array}{c}1^{\text {st }} \\
\text { cluster }\end{array}$ & $\begin{array}{c}2^{\text {nd }} \\
\text { cluster }\end{array}$ & $\begin{array}{c}3^{\text {rd }} \\
\text { cluster }\end{array}$ & $\begin{array}{c}\text { Avg. } \\
1^{\text {st }}+2^{\text {nd }}+3^{\text {rd }} \\
\text { clusters }\end{array}$ & $\begin{array}{c}1^{\text {st }} \\
\text { cluster }\end{array}$ & $\begin{array}{c}2^{\text {nd }} \\
\text { cluster }\end{array}$ & $\begin{array}{c}3^{\text {rd }} \\
\text { cluster }\end{array}$ & $\begin{array}{c}\text { Avg. } \\
1^{\text {st }}+2^{\text {nd }}+3^{\text {rd }} \\
\text { clusters }\end{array}$ \\
\hline & & \multicolumn{4}{|c|}{ First season (2014) } & \multicolumn{4}{|c|}{ Second season (2015) } \\
\hline \multirow{6}{*}{ Bare plants } & Control & $41.67 \mathrm{de}$ & $83.00 \mathrm{a}$ & $56.67 \mathrm{c}$ & $59.89 \mathrm{~d}$ & $45.83 \mathrm{~d}$ & $74.50 \mathrm{~cd}$ & $52.50 \mathrm{de}$ & $57.61 \mathrm{~g}$ \\
\hline & $\mathbf{C a}+\mathbf{B}$ & $87.50 \mathrm{ab}$ & $50.00 \mathrm{a}$ & $58.33 \mathrm{c}$ & $65.28 \mathrm{~cd}$ & $93.75 \mathrm{ab}$ & $55.00 \mathrm{e}$ & $54.17 \mathrm{~d}$ & $67.64 f$ \\
\hline & GA3 & $100.00 \mathrm{a}$ & $65.00 \mathrm{a}$ & $90.00 \mathrm{ab}$ & $85.00 \mathrm{ab}$ & $100.00 \mathrm{a}$ & $72.50 \mathrm{~cd}$ & $85.00 \mathrm{bc}$ & $85.83 b c$ \\
\hline & IAA & $80.00 \mathrm{~b}$ & $55.00 \mathrm{a}$ & $75.00 \mathrm{bc}$ & $70.00 \mathrm{~cd}$ & $90.00 \mathrm{~b}$ & $52.50 \mathrm{e}$ & $87.50 \mathrm{bc}$ & $76.67 \mathrm{de}$ \\
\hline & $\mathbf{G A 3}+\mathrm{Ca}+\mathrm{B}$ & $90.00 \mathrm{ab}$ & $77.50 \mathrm{a}$ & $87.50 \mathrm{ab}$ & $85.00 \mathrm{ab}$ & $85.00 \mathrm{~b}$ & $76.25 \mathrm{~cd}$ & $81.25 \mathrm{c}$ & $80.83 \mathrm{~cd}$ \\
\hline & $\mathbf{I A A}+\mathbf{C a}+\mathbf{B}$ & $63.33 \mathrm{c}$ & $100.0 \mathrm{a}$ & $65.00 \mathrm{c}$ & $76.11 b c$ & $61.67 \mathrm{c}$ & $100.00 \mathrm{a}$ & $57.50 \mathrm{~d}$ & $73.06 \mathrm{ef}$ \\
\hline \multirow{6}{*}{$\begin{array}{c}\text { Floating } \\
\text { cover (Agryl) }\end{array}$} & Control & $36.67 \mathrm{e}$ & $54.00 \mathrm{a}$ & $35.00 \mathrm{~d}$ & $41.89 \mathrm{e}$ & $35.00 \mathrm{e}$ & $67.00 \mathrm{~d}$ & $42.50 \mathrm{e}$ & $48.17 \mathrm{~h}$ \\
\hline & $\mathbf{C a}+\mathbf{B}$ & $55.00 \mathrm{~cd}$ & $87.50 \mathrm{a}$ & $32.50 \mathrm{~d}$ & $58.33 \mathrm{~d}$ & $52.50 \mathrm{~d}$ & $81.25 b c$ & $28.75 f$ & $54.17 \mathrm{gh}$ \\
\hline & GA3 & $100.00 \mathrm{a}$ & $83.00 \mathrm{a}$ & $100.00 \mathrm{a}$ & $94.33 \mathrm{a}$ & $100.00 \mathrm{a}$ & $74.50 \mathrm{~cd}$ & $100.00 \mathrm{a}$ & $91.50 \mathrm{ab}$ \\
\hline & IAA & $90.00 \mathrm{ab}$ & $93.75 \mathrm{a}$ & $91.50 \mathrm{ab}$ & $91.75 \mathrm{a}$ & $85.00 \mathrm{~b}$ & $90.63 \mathrm{ab}$ & $95.75 \mathrm{ab}$ & $90.46 b$ \\
\hline & $\mathbf{G A 3}+\mathrm{Ca}+\mathrm{B}$ & $95.45 \mathrm{ab}$ & $87.50 \mathrm{a}$ & $91.50 \mathrm{ab}$ & $91.48 \mathrm{a}$ & $93.18 \mathrm{ab}$ & $93.75 \mathrm{ab}$ & $87.25 \mathrm{bc}$ & $91.39 \mathrm{ab}$ \\
\hline & $\mathbf{I A A}+\mathbf{C a}+\mathbf{B}$ & $87.50 \mathrm{ab}$ & $100.00 \mathrm{a}$ & $100.00 \mathrm{a}$ & $95.83 \mathrm{a}$ & $93.75 \mathrm{ab}$ & $100.00 \mathrm{a}$ & $100.00 \mathrm{a}$ & $97.92 \mathrm{a}$ \\
\hline
\end{tabular}

Table (10): Effect of floating cover (Agryl) and fruit setting substances on yield of tomato plant

\begin{tabular}{|c|c|c|c|c|c|c|c|c|c|c|}
\hline \multirow{3}{*}{ Treatments } & \multicolumn{2}{|c|}{ Marketable yield } & \multirow{2}{*}{$\begin{array}{c}\text { Unmar- } \\
\text { ketable } \\
\text { yield } \\
\text { (ton/fed.) }\end{array}$} & \multirow{2}{*}{$\begin{array}{c}\text { Total } \\
\text { yield } \\
\text { (ton/fed.) }\end{array}$} & \multirow{2}{*}{$\begin{array}{l}\text { No. } \\
\text { seeds/ } \\
\text { fruit }\end{array}$} & \multicolumn{2}{|c|}{ Marketable yield } & \multirow{2}{*}{$\begin{array}{l}\text { Unmar- } \\
\text { ketable } \\
\text { yield } \\
\text { (ton/fed.) }\end{array}$} & \multirow{2}{*}{$\begin{array}{c}\text { Total } \\
\text { yield } \\
\text { (ton/fed.) }\end{array}$} & \multirow{2}{*}{$\begin{array}{c}\text { No. } \\
\text { seeds/ } \\
\text { fruit }\end{array}$} \\
\hline & $\begin{array}{c}\text { Yield/plant } \\
\text { (g) }\end{array}$ & $\begin{array}{c}\text { Yield } \\
\text { (ton/fed.) }\end{array}$ & & & & $\begin{array}{c}\text { Yield/plant } \\
\text { (g) }\end{array}$ & $\begin{array}{c}\text { Yield } \\
\text { (ton/fed.) }\end{array}$ & & & \\
\hline & \multicolumn{5}{|c|}{ First season (2014) } & \multicolumn{5}{|c|}{ Second season (2015) } \\
\hline Bare plants & $2611.0 \mathrm{a}$ & $14.01 \mathrm{a}$ & $0.282 \mathrm{a}$ & $14.272 \mathrm{a}$ & $155.01 \mathrm{a}$ & $2852.0 \mathrm{a}$ & $13.38 \mathrm{a}$ & $0.349 \mathrm{a}$ & $13.729 a$ & $149.23 \mathrm{a}$ \\
\hline $\begin{array}{c}\text { Floating } \\
\text { cover (Agryl) }\end{array}$ & $2305.0 \mathrm{~b}$ & $10.90 \mathrm{~b}$ & $0.117 \mathrm{~b}$ & $11.017 \mathrm{~b}$ & $60.33 b$ & $2271.0 \mathrm{~b}$ & $10.70 \mathrm{~b}$ & $0.125 b$ & $10.805 b$ & $60.63 b$ \\
\hline Control & $2196 d$ & $10.54 \mathrm{e}$ & $0.107 \mathrm{~d}$ & $10.647 \mathrm{~d}$ & $81.50 \mathrm{c}$ & $2073 d$ & $9.77 \mathrm{c}$ & $0.117 \mathrm{e}$ & $9.889 \mathrm{~d}$ & $78.50 \mathrm{c}$ \\
\hline $\mathbf{C a}+\mathbf{B}$ & $2740 \mathrm{~b}$ & $13.14 b c$ & $0.139 \mathrm{c}$ & $13.279 \mathrm{ab}$ & $108.75 b$ & $2724 a b$ & $12.86 \mathrm{ab}$ & $0.158 \mathrm{~d}$ & $13.018 \mathrm{ab}$ & $103.15 b$ \\
\hline $\mathbf{G A}_{3}$ & $2531 \mathrm{c}$ & $12.15 \mathrm{~cd}$ & $0.170 \mathrm{~b}$ & $12.320 \mathrm{bc}$ & $95.25 \mathrm{bc}$ & $2460 \mathrm{c}$ & $11.35 \mathrm{~b}$ & $0.257 \mathrm{bc}$ & $11.604 \mathrm{c}$ & $106.08 b$ \\
\hline IAA & $2560 \mathrm{c}$ & $11.39 \mathrm{~d}$ & $0.394 \mathrm{a}$ & $11.784 \mathrm{~cd}$ & $108.28 b$ & $2480 \mathrm{bc}$ & $11.90 \mathrm{~b}$ & $0.376 \mathrm{a}$ & $12.276 \mathrm{bc}$ & $93.30 \mathrm{bc}$ \\
\hline $\mathbf{G A}_{3}+\mathbf{C a}+\mathbf{B}$ & $2757 b$ & $13.22 \mathrm{ab}$ & $0.204 \mathrm{~b}$ & $13.424 \mathrm{ab}$ & $109.00 \mathrm{~b}$ & $2722 \mathrm{ab}$ & $12.75 \mathrm{ab}$ & $0.292 b$ & $13.042 \mathrm{ab}$ & $104.16 \mathrm{~b}$ \\
\hline $\mathbf{I A A}+\mathbf{C a}+\mathbf{B}$ & $3154 a$ & $14.32 \mathrm{a}$ & $0.183 b$ & $14.503 \mathrm{a}$ & $143.30 \mathrm{a}$ & $2912 \mathrm{a}$ & $13.61 \mathrm{a}$ & $0.222 \mathrm{c}$ & $13.832 \mathrm{a}$ & $136.40 \mathrm{a}$ \\
\hline
\end{tabular}


Table (11): Effect of interaction between floating cover and fruit setting substances on yield of tomato plant

\begin{tabular}{|c|c|c|c|c|c|c|c|c|c|c|c|}
\hline \multirow{2}{*}{\multicolumn{2}{|c|}{ Treatments }} & \multicolumn{2}{|c|}{ Marketable yield } & \multirow{2}{*}{$\begin{array}{c}\text { Unmarket- } \\
\text { able yield } \\
\text { (ton/fed.) }\end{array}$} & \multirow{2}{*}{$\begin{array}{c}\text { Total yield } \\
\text { (ton/fed.) }\end{array}$} & \multirow{3}{*}{$\begin{array}{l}\text { No. } \\
\text { seeds/ } \\
\text { fruit }\end{array}$} & \multicolumn{2}{|c|}{ Marketable yield } & \multirow{2}{*}{$\begin{array}{l}\text { Unmarket- } \\
\text { able yield } \\
\text { (ton/fed.) }\end{array}$} & \multirow{2}{*}{$\begin{array}{c}\text { Total yield } \\
\text { (ton/fed.) }\end{array}$} & \multirow{2}{*}{$\begin{array}{c}\text { No. } \\
\text { seeds/ } \\
\text { fruit }\end{array}$} \\
\hline & & $\begin{array}{c}\text { Yield/ } \\
\text { Plant (g) }\end{array}$ & $\begin{array}{c}\text { Yield } \\
\text { (ton/fed.) }\end{array}$ & & & & $\begin{array}{c}\text { Yield/ } \\
\text { Plant (g) }\end{array}$ & $\begin{array}{c}\text { Yield } \\
\text { (ton/fed.) } \\
\end{array}$ & & & \\
\hline & & \multicolumn{4}{|c|}{ First season (2014) } & & \multicolumn{5}{|c|}{ Second season $(2015)$} \\
\hline \multirow{6}{*}{ Bare plants } & Control & $2358 \mathrm{c}$ & $11.32 \mathrm{~cd}$ & $0.107 \mathrm{c}$ & $11.427 \mathrm{~d}$ & $137.00 \mathrm{~b}$ & $2209 \mathrm{ef}$ & $10.24 \mathrm{de}$ & $0.120 \mathrm{f}$ & 10.360 & $122.00 \mathrm{~b}$ \\
\hline & $\mathbf{C a}+\mathbf{B}$ & $3278 \mathrm{a}$ & $15.72 \mathrm{a}$ & $0.150 \mathrm{bc}$ & $15.870 \mathrm{a}$ & $140.00 \mathrm{~b}$ & $2890 \mathrm{bc}$ & $13.87 \mathrm{~b}$ & $0.181 \mathrm{e}$ & $14.051 \mathrm{~b}$ & $132.50 \mathrm{~b}$ \\
\hline & $\mathbf{G A}_{3}$ & $2893 b$ & $13.89 \mathrm{~b}$ & $0.236 b c$ & $14.126 b$ & $163.00 \mathrm{a}$ & $2725 \mathrm{bcd}$ & $12.16 \mathrm{c}$ & $0.404 \mathrm{c}$ & $12.564 \mathrm{c}$ & $166.50 \mathrm{a}$ \\
\hline & IAA & $2872 b$ & $12.01 \mathrm{c}$ & $0.641 \mathrm{a}$ & $12.651 \mathrm{c}$ & $151.56 \mathrm{ab}$ & $2919 b$ & $14.01 \mathrm{~b}$ & $0.604 \mathrm{a}$ & $14.614 b$ & $145.60 \mathrm{a}$ \\
\hline & $\mathbf{G A}_{3}+\mathbf{C a}+\mathbf{B}$ & $3207 a$ & $15.37 \mathrm{a}$ & $0.304 \mathrm{~b}$ & $15.674 \mathrm{a}$ & $169.00 \mathrm{a}$ & $3154 a$ & $14.90 \mathrm{a}$ & $0.473 b$ & $15.373 \mathrm{a}$ & $162.00 \mathrm{a}$ \\
\hline & $\mathbf{I A A}+\mathbf{C a}+\mathbf{B}$ & $3418 \mathrm{a}$ & $15.79 \mathrm{a}$ & $0.353 b c$ & $16.143 \mathrm{a}$ & $169.50 \mathrm{a}$ & $3215 \mathrm{a}$ & $15.13 \mathrm{a}$ & $0.121 \mathrm{~d}$ & $15.251 \mathrm{a}$ & $166.80 \mathrm{a}$ \\
\hline \multirow{6}{*}{$\begin{array}{c}\text { Floating cover } \\
\text { (Agryl) }\end{array}$} & Control & $2035 \mathrm{e}$ & $9.77 \mathrm{e}$ & $0.107 \mathrm{c}$ & $9.877 \mathrm{f}$ & $26.00 \mathrm{e}$ & $1938 f$ & $9.30 \mathrm{f}$ & $0.114 \mathrm{f}$ & $9.414 \mathrm{e}$ & $35.00 \mathrm{f}$ \\
\hline & $\mathbf{C a}+\mathbf{B}$ & $2202 d$ & $10.56 \mathrm{de}$ & $0.128 b c$ & $10.688 \mathrm{def}$ & $77.50 \mathrm{~d}$ & $2558 \mathrm{~d}$ & $11.860 \mathrm{c}$ & $0.135 f$ & $11.995 \mathrm{c}$ & $73.85 \mathrm{~d}$ \\
\hline & $\mathbf{G A}_{3}$ & 2170de & $10.41 \mathrm{de}$ & $0.104 \mathrm{c}$ & $10.514 \mathrm{ef}$ & $27.50 \mathrm{e}$ & $2195 \mathrm{ef}$ & $10.54 \mathrm{de}$ & $0.110 \mathrm{f}$ & $10.650 \mathrm{~d}$ & $45.67 \mathrm{ef}$ \\
\hline & IAA & $2248 d$ & $10.77 \mathrm{~d}$ & $0.147 b c$ & $10.917 \mathrm{de}$ & $65.00 \mathrm{~d}$ & $2041 \mathrm{ef}$ & $9.79 \mathrm{ef}$ & $0.148 \mathrm{ef}$ & $9.938 \mathrm{de}$ & $41.00 \mathrm{e}$ \\
\hline & $\mathbf{G A}_{3}+\mathbf{C a}+\mathbf{B}$ & $2307 \mathrm{~cd}$ & $11.07 \mathrm{~d}$ & $0.103 \mathrm{c}$ & $11.173 \mathrm{de}$ & $49.00 \mathrm{e}$ & $2290 \mathrm{e}$ & $10.61 d$ & $0.111 \mathrm{f}$ & $10.721 d$ & 46.33ef \\
\hline & $\mathbf{I A A}+\mathbf{C a}+\mathbf{B}$ & $2873 b$ & $12.86 \mathrm{c}$ & $0.114 \mathrm{c}$ & $12.974 \mathrm{c}$ & $117.00 \mathrm{c}$ & $2609 \mathrm{~cd}$ & $12.09 \mathrm{c}$ & $0.132 \mathrm{f}$ & $12.222 \mathrm{c}$ & $106.00 c$ \\
\hline
\end{tabular}

Values having the same alphabetical letter (s) did not significantly differ at 0.05 level of significance according to Duncan's multiple range test 


\section{CONCLUSION}

The performance of tomato plants under high temperature stress (which grown under floating covers) was improved by spraying some substances as $\mathrm{Ca}+\mathrm{B}$, IAA, or $\mathrm{GA}_{3}$ which enhanced the plant growth, proline concentration in leaves and fruit setting (\%). On the other hand, total chlorophyll, marketable yield per plant and number of seeds/fruit which expressed as the viability of pollen grains and fruit size were decreased under high temperatures stress.

\section{REFERENCES}

Aggarwal, M., S. Sharma, N. Kaur, D. Patahnia, K. Bhandhari, N. kausha, R. kaur, K. Singh, A. Srivastava and H. Nayyar (2011). Exogenous proline application reduces phytotoxic effects of selenium by minimising oxidative stress and improves growth in bean (Phaseolus vulgaris L.) seedlings. Biol. Trace Element Res., 140(3): 354-367.

Al-Shihi, A. A., A. M. Al-Said, F. A. Al-Said, U. E. Ammara and M. L. Deadman (2016). Optimising the duration of floating row cover period to minimise the incidence of tomato yellow leaf curl disease and maximise yield of tomato. Ann. Appl. Biol., 168: 328-336.

Asad A., E. P. C. Blamey and D. G. Edward (2003). Effects of boron foliar applications on vegetative and reproductive growth of sunflower. Ann. Bot., 92: 565-570.

Bates, L. S., R. P. Waldren and I. D. Tare (1973). Rapid determination of free proline for water-stress studies. Plant and Soil, 39: 205-207.

Berlinger M. J., R. A. J. Taylor, S. Lebiush-Mordechi, S. Shalhevet and I. Spharim (2002). Efficiency of insect exclusion screens for preventing whitefly transmission of tomato yellow leaf curl virus of tomatoes in Israel. Bulletin of Entomological Research, 92: 367373.

de Jong, M., C. Mariani and W. H. Vriezen (2009). The role of auxin and gibberellin in tomato fruit set. J. Exp. Bot., 60(5): 1523-1532.

Desouky, I. M., L. F. Haggog, M. M. M.Abd-ElMigeed, Y. F. M.Kishk and E. S. El-Hadi (2009). Effect of boron and calcium nutrients sprayson fruit set, oil content and oil quality of some olive cultivars. World J. Agric. Sci., 5: $180-185$.

Duncan, D. B. (1955). Multiple rang and multiple F test. Biometrics, 11: 1-42.

El-Sherbeny, M. R. and J. A. T. Da Silva (2013). Foliar treatment with proline and tyrosine affect the growth and yield of beetroot and some pigments in beetroot leaves. J. Hort. Res., 21(2): 95-99.

Gordon, G. G. (2006). The effects of color plastic mulches and row covers on the growth and yield of okra and summer squash. M.Sc. Thesis, Auburn University, 74 pp.

Haque, M. E., A. K. Paul and J. R. Sarker (2011). Effect of nitrogen and boronon the growth and yield of tomato (Lycopersicon esculentum M.). Inter. J. Bio-resource and Stress Manag., 2(3): 277-282.

Hare, P. D., W. A. Cress and J. van Staden (2003). A regulatory role for proline metabolism in stimulating Arabidopsis thaliana seed germination. Plant Growth Regul., 39: 494500 .

Hopkins, W. G. and N. P. A. Hüner (2009). Introduction to Plant Physiology. $4^{\text {th }}$ ed. John Wiley \& Sons, Inc. USA., 503 pp.

Hu, W. H., Y. A. Xiao, J. J. Zeng and X. H. Hu (2010). Photosynthesis, respiration and antioxidant enzymes in pepper leaves under drought and heat stresses. Biologia Plantarum, 54(4): 761765.

Kafizadeh, N., J. Carapetian and K. M. Kalantari (2008). Effect of heat stress on pollen viability and pollen tube growth in pepper. Res. J. Biol. Sci., 3(10): 1159-1162.

Kaushal N., K. Gupta, K. Bhandhari, S. Kumar, P. Thakur and H. Nayyar (2011). Proline induces heat tolerance in chickpea (Cicer arietinum L.) plants by protecting vital enzymes of carbon and antioxidative metabolism. Physiol. Mol. Biol. Plants, 17(3): 203-213.

Khan, M. M. A., C. Gautam, F. Mohammad, M. H. Siddiqui, M. Naeem and M. N. Khan (2006). Effect of Gebberellic acid spray on performance of tomato. Turk. J. Biol., 30: 1116.

Leah, K. and B. Aloni (2002). Fruktokinase and hexokinase from pollen grains of bell pepper (Capsicum annuum L.): possible role in pollen germination under conditions of high temperature and $\mathrm{CO}_{2}$ enrichment. Ann. Bot., 90: 607-612.

Maggio, A., S. Miyazaki, P. Veronese, T. Fujita, J. Ibeas, B. Damsz, M. L. Narasimhan, P. M. Hasegawa, R. J. Joly and R. A. Bressan (2002). Does proline accumulation play an active role in stress-induced growth reduction? The Plant Journal, 31(6): 699-712.

Mestre,T. C., F. Garcia-Sanchez, F. Rubio, V. Martinez and R. M. Rivero (2012). Glutathione homeostasis as an important and novel factor controlling blossom-end rot development in calcium-deficient tomato fruits. J. Plant Physiol., 169: 1719- 1727.

Ördog, V. (2011). Plant Physiology. Az Agrármérnöki MSc szak tananyagfejlesztése, TÁMOP4.1.2-08/1/A-2009-0010 projekt 115pp.

Pattison, R. J., F. Csukasi and C. Catala (2014). Mechanisms regulating auxin action during fruit development. Physiological Plantarum, 151(1): 62-72.

Peet, M. M., D. H. Willits and R. Gardner (1997). Response of ovule development and post- 
pollenproduction processes in male-sterile tomatoes tochronic, sub-acute high temperature stress. J. Exp. Bot., 48(306): 101111.

Pressman, E., M. M. Peet and M. Pharr (2002). The effect of heat stress on tomato pollen characteristics is associated with changes in carbohydrate concentration in the developing anther. Ann. Bot., 90: 631-636.

Rab, A. and I. Haq (2012). Foliar application of calcium chloride and borax influences plant growth, yield, and quality of tomato (Lycopersicon esculentum Mill.) fruit. Turk. J. Agric. For., 36: 695-701.

Saidi, M., E. O. Gogo, F. M. Itulya, T. Martin and M. Ngouajio (2013). Microclimate modification using eco-friendly nets and floating row covers improves tomato (Lycopersicon esculentum) yield and quality for small holder farmers in East Africa. Agric. Sci., 4(11): 577-584.

Smit, J. N. and N. J. J. Combrink (2005). Pollination and yield of winter-grown greenhouse tomatoes as affected by boron nutrition, cluster vibration and relative humidity. South Afr. J. Plant Soil, 22: 110-115.
Snedecor, G. W. and W. G. Cochran (1980). Statistical Methods $7^{\text {th }}$ ed. Iowa State Univ., Press. Ames. Iowa, USA.

Srivastava, P. C. and U. C. Gupta (1996). Trace Elements in Crop Production. Science Publisher Inc., pp 355.

Székely, G., E. Abrahám, A. Cséplo, G. Rigó, L. Zsigmond, J. Csiszár, F. Ayaydin, N. Strizhov, J. Jásik, E. Schmelzer, C. Koncz and L. Szabados (2008). Duplicated P5CS genes of Arabidopsis play distinct roles in stress regulation and developmental control of proline biosynthesis. Plant J., 53(1): 11-28.

Taber, H. G. and A. Webb (2008). Row covers for early tomato production. Iowa State Research Farm Progress Reports, Pp 515.

Verbruggen, N. and C. Hermans (2008). Proline accumulation in plants: a review. Amino Acids, 35(4): 753-759.

Zhou, R., K. H. Kjær, E. Rosenqvist, X. Yu, Z. Wu and C.-O. Ottosen (2016). Physiological response to heat stress during seedling and anthesis stage in tomato genotypes differing in heat tolerance. J. Agron. Crop Sci., 63(1): 68-80.

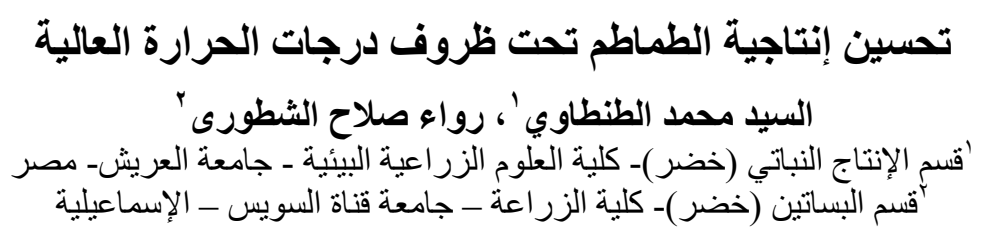

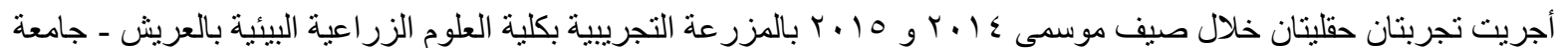

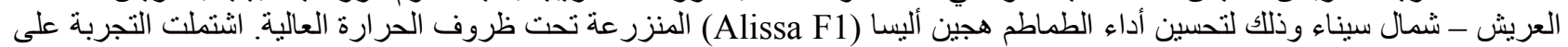

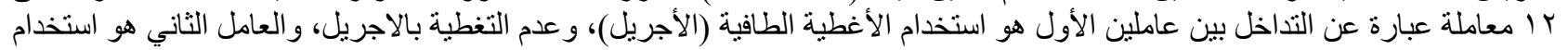

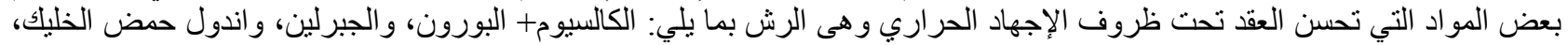

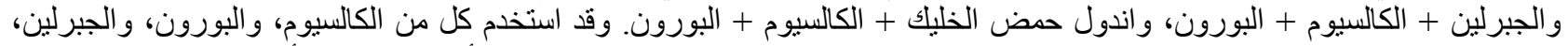

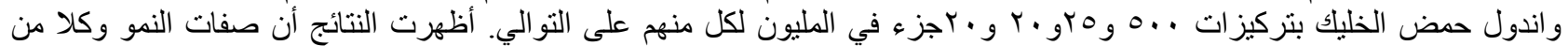

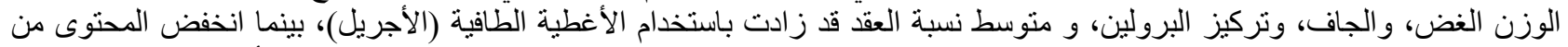

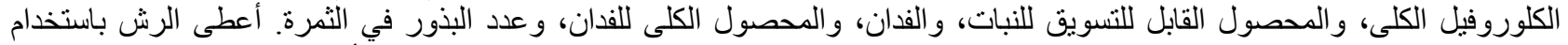

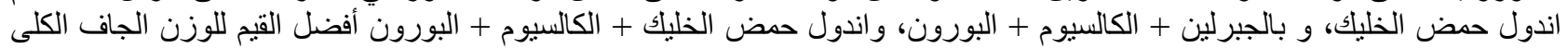

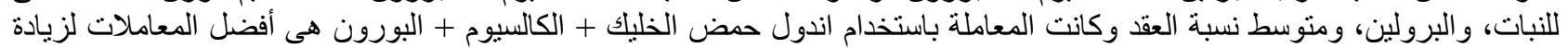

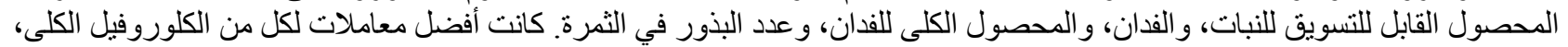

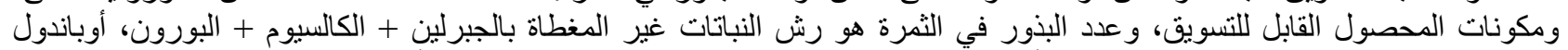

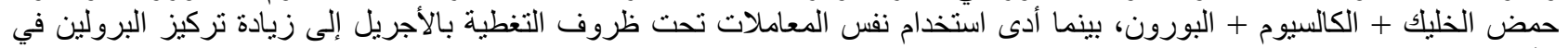

